\title{
Reductive Iron Assimilation and Intracellular Siderophores Assist Extracellular Siderophore-Driven Iron Homeostasis and Virulence
}

\author{
Bradford J. Condon, ${ }^{1}$ Shinichi Oide, ${ }^{1,2}$ Donna M. Gibson, ${ }^{3}$ Stuart B. Krasnoff, ${ }^{3}$ and B. Gillian Turgeon ${ }^{1}$ \\ ${ }^{1}$ Plant Pathology \& Plant-Microbe Biology, Cornell University, Ithaca, NY 14853, U.S.A.; ${ }^{2}$ The Research Institute of Innovative \\ Technology for the Earth (RITE), 9-2, Kizugawadai, Kizugawa-Shi, Kyoto, Japan; ${ }^{3}$ Biological Integrated Pest Management Unit, \\ USDA-ARS, Robert W. Holley Center, Ithaca, NY, 14853, U.S.A.
}

Submitted 7 November 2013. Accepted 10 April 2014.

Iron is an essential nutrient and prudent iron acquisition and management are key traits of a successful pathogen. Fungi use nonribosomally synthesized secreted iron chelators (siderophores) or reductive iron assimilation (RIA) mechanisms to acquire iron in a high affinity manner. Previous studies with the maize pathogen Cochliobolus heterostrophus identified two genes, NPS2 and NPS6, encoding different nonribosomal peptide synthetases responsible for biosynthesis of intra- and extracellular siderophores, respectively. Deletion of NPS6 results in loss of extracellular siderophore biosynthesis, attenuated virulence, hypersensitivity to oxidative and iron-depletion stress, and reduced asexual sporulation, while nps 2 mutants are phenotypically wild type in all of these traits but defective in sexual spore development when NPS2 is missing from both mating partners. Here, it is reported that nps2nps6 mutants have more severe phenotypes than both nps 2 and nps 6 single mutants. In contrast, mutants lacking the FTR1 or FET3 genes encoding the permease and ferroxidase components, respectively, of the alternate RIA system, are like wild type in all of the above phenotypes. However, without supplemental iron, combinatorial nps6ftr1 and nps2nps6ftr1 mutants are less virulent, are reduced in growth, and are less able to combat oxidative stress and to sporulate asexually, compared with nps 6 mutants alone. These findings demonstrate that, while the role of RIA in metabolism and virulence is overshadowed by that of extracellular siderophores as a high-affinity iron acquisition mechanism in $C$. heterostrophus, it functions as a critical backup for the fungus.

Iron is a vital nutrient and its redox activity has a central role in many enzymatic reactions (Kaplan and Kaplan 2009). Bioavailable iron, however, is limited, as iron is largely insoluble in the presence of oxygen (Haas et al. 2008; Lindsay and Schwab 1982). Iron's capacity as a redox mediator can also generate reactive oxygen species (ROS) and cause subsequent cellular damage via the Fenton reaction (Fenton 1894; Winterbourn 1995). All organisms must, therefore, balance iron acquisition,

Corresponding author: B. G. Turgeon; E-mail: bgt1@ cornell.edu

* The $e$-Xtra logo stands for "electronic extra" and indicates that seven supplementary figures and one supplementary table are published online and that Figures 1, 2, 6, 8, and 9 appear in color online.

(C) 2014 The American Phytopathological Society chelation, and storage with detoxification of the oxygen radicals free iron can produce (Kaplan and Kaplan 2009).

Fungi, including fungal phytopathogens, have two known methods for high-affinity iron acquisition, i.e., reductive iron assimilation (RIA) and siderophore assisted (Haas et al. 2008; Johnson 2008). RIA, originally characterized in Saccharomyces cerevisiae, is an extracellular membrane-bound process whereby ferric iron is reduced by ferric reductase Fer1 and then oxidized by the iron multicopper oxidase Fet3 while being transported across the plasma membrane by the high-affinity iron permease Ftr1 (Kosman 2003, 2010). Ftr1 and Fet3 are intimately linked and require one another to co-localize to the plasma membrane (Singh et al. 2006; Stearman et al. 1996). Alternatively, fungi can utilize siderophores, small cyclic peptides produced by nonribosomal peptide synthetases (NRPS) (Schwarzer et al. 2003), which are found only in fungi and bacteria and absent from animals and plants (Finking and Marahiel 2004).

Iron is important for host-microbe interactions, as pathogens must gain access to host-chelated iron to survive. This paradigm is well-established for fungal pathogens of humans. Aspergillus fumigatus, for example, requires both intra- and extracellular siderophores for full virulence on a variety of hosts (Hissen et al. 2005; Schrettl et al. 2004, 2007). Conversely, deletion of the RIA-affiliated permease gene ftrA in A. fumigatus has no effect on virulence in mice (Schrettl et al. 2004). Double deletion mutants of the ftrA gene and the sidA gene, which encodes $\mathrm{N}^{5}$-ornithine-monooxygenase essential for biosynthesis of both intraand extracellular siderophores, are unable to grow on most media without siderophore (ferricrocin) supplementation, demonstrating that the RIA mechanism does contribute to iron acquisition in the absence of siderophores (Schrettl et al. 2004).

Early studies of iron acquisition by plant pathogens found that the biotrophs Ustilago maydis and Microbotryum violaceum do not require siderophores for virulence, suggesting iron acquisition was not vital for phytopathogens (Birch and Ruddat 2005; Mei et al. 1993). Later studies demonstrated that $U$. maydis instead requires RIA, as RIA mutants are strongly reduced in virulence (Eichhorn et al. 2006). In the necrotroph Fusarium graminearum, RIA (iron permease) mutants are like wild type (WT) for both growth and virulence (Park et al. 2006). These mutants show increased SIDA expression comparable to that seen in iron-depleted WT strains, however, suggesting RIA is, in fact, utilized to acquire iron in F. graminearum (Park et al. 2006).

A positive role for siderophores in fungal pathogenesis to plants was demonstrated when Cochliobolus heterostrophus, 
Cochliobolus miyabeanus, Alternaria brassicicola, and F. graminearum mutants deleted for the NPS6 gene, encoding an NRPS responsible for biosynthesis of the extracellular siderophore coprogen, all showed reduced virulence on their respective hosts (corn, rice, Arabidopsis, wheat) (Oide et al. 2006). Deletion of NPS6 affects more than virulence in all of these species; it also results in hypersensitivity to ROS and hypersensitivity to iron depletion (Lee et al. 2005; Oide et al. 2006).

In contrast, deletion of $C$. heterostrophus NPS2, encoding an NRPS for biosynthesis of the intracellular siderophore ferricrocin, responsible for iron storage, impairs sexual reproduction but not virulence. The nps 2 mutants cannot make ascospores in homozygous $n p s 2 \times n p s 2$ crosses, although these crosses form pseudothecia and are hermaphroditic like WT strains (Oide et al. 2007). A similar sexual development phenotype was found for homothallic F. graminearum; deletion of the NPS2 ortholog results in loss of ferricrocin biosynthesis and defects in ascospore development (Oide et al. 2007).

Pathogens apparently rely on either RIA or siderophores as their primary means of iron acquisition but not both. The underlying mechanism for the reduction of virulence in either RIA or siderophore mutants is unclear, however. $C$. heterostrophus nps6 mutants are sensitive to both oxidative and iron stress, both of which could be encountered in planta (Oide et al. 2006). Prussian blue staining has shown that $\mathrm{Fe}^{3+}$ accumulates at infection sites of the biotroph Blumeria graminis $\mathrm{f}$. $\mathrm{sp}$. tritici on various monocots (Liu et al. 2007). For the necrotroph Botrytis cinerea, virulence increases in synchrony with the intensity of ROS, contributed both by the host and the fungus (Temme and Tudzynski 2009). Similarly, starving Arabidopsis for iron by washing roots with an iron chelator reduces susceptibility to $B$. cinerea. Increased fungal siderophore production suggested this reduction in disease was related to iron starvation as opposed to another mechanism such as antimicrobial activity (Kieu et al. 2012).

Additionally, ROS link iron to many biological processes, such as sexual development, signal transduction, and stress responses in fungi as well as plants (Aguirre et al. 2005; Foyer and Noctor 2005). Plant-generated ROS mediate activation of defense genes or additional defenses by redox control of transcription factors or through other signaling components, such as phosphorylation cascades (Kotchoni and Gachomo 2006; Mou et al. 2003). The sexual dysfunction phenotype of $C$. heterostrophus nps 2 mutants could be a result of impaired iron or ROS status (Oide et al. 2007).

The extent to which RIA contributes to virulence of the necrotroph $C$. heterostrophus is unknown. Furthermore, RIA and siderophore polymutants have been reported only in Aspergillus species and no differentiation between the absence of extracellular versus intracellular siderophores was made. To address these questions, we constructed RIA mutant strains by deleting the high-affinity permease FTRl and generated combinatorial nps2ftrl, nps6ftrl, and nps2nps6ftrl mutants. We analyzed the phenotypes of these mutants with regard to nutrition, iron and oxidative stress, and virulence and provide evidence for a secondary role of RIA in C. heterostrophus.

\section{RESULTS}

\section{Identification of RIA mutants.}

BLAST searches using the $S$. cerevisiae proteins as a query identified FTRI (Joint Genome Institute [JGI] identification number $104817,1,778 \mathrm{bp}$ ) and FET3 (JGI identification number $104814,2,030 \mathrm{bp}$ ) orthologs separated by 1,778 bp in $C$. heterostrophus strains $\mathrm{C} 4$ and $\mathrm{C} 5$. This region was identical in the two strains except for two single nucleotide polymorphisms that do not alter the coding sequence of either FTRI or
FET3. Individual FTRI and FET3 deletion mutants were generated in strain $\mathrm{C} 4$. Mutants with genes deleted and replaced by selectable marker $H y g B$ were verified using diagnostic polymerase chain reaction (PCR) primers as described below and in Supplementary Figure S1. All gene deletion and verification primers are listed in Supplementary Table $S 1$.

None of the RIA mutants (fet $3, f t r l$, fet $3 f t r l$; Table 1) was different from WT with respect to growth, sexual and asexual development, sensitivity to oxidative and iron stress, or virulence.

\section{Identification of high-affinity iron acquisition and iron storage polymutants.}

Combinatorial mutants lacking ability to produce intracellular siderophores (nps2), extracellular siderophores (nps6), or RIA ( $f t r l, f e t 3, f t r l f e t 3$ ) components were generated by crossing single or single and double mutants as described below. The nps2nps6, nps6ftrl, nps2ftr1, nps6fet 3 , nps6fet $3 f t r 1$, and nps2nps6ftrl (the latter shown in Figure 1) mutants were collected in this way and were confirmed by PCR with the primer combinations used to identify the original mutants among transformants. All strains used in this study are listed in Table 1 and all crosses are listed in Table 2.

\section{Iron combinatorial mutants require supplemental iron for growth.}

Previously, we showed that mutants lacking extracellular (nps6) or intracellular (nps2) siderophores grow at WT rates on complete medium with xylose (CMX) (Oide et al. 2006; 2007). The ftrl, fet 3 , and fet $3 f t r l$ RIA mutants generated here also displayed WT morphology and growth rates. Double mutants lacking both NPS2 and NPS6 also grew like WT (Fig. 2). In contrast, all mutants lacking both extracellular siderophores and RIA (nps6ftrl, nps6fet3, nps6fet3ftrl) grew at a reduced rate on CMX and were less pigmented than WT strains (Fig. 2 ). Growth and pigmentation of these mutants could be restored to WT levels by providing $100 \mu \mathrm{M}$ supplemental ferric citrate (Fig. 2). The nps2nps6ftrl triple mutants, lacking not only extracellular siderophore and RIA iron acquisition mechanisms but also intracellular siderophores and, thus, the ability to store iron, were almost completely unable to grow on CMX and did not sporulate (Fig. 2). Even with supplemental iron, close examination of colony morphology revealed significant alterations compared with nps6ftrl.

On minimal medium (MM), the reduced growth and pigmentation phenotype of nps6ftrl, nps6fet3, and nps6fet $3 f t r 1$ mutants was even more pronounced (Fig. 2). These mutants were all similarly reduced in growth, which suggests that deleting either FTR1 or FET3 is sufficient to disrupt RIA. Subsequently, most experiments reported in this manuscript used ftrl mutants to represent RIA deficiency. The nps2nps6 and nps2ftrl mutants grew like WT strains on MM (Fig. 2); however, nps $2 n p s 6$ mutants, like nps6 mutants, were reduced in pigmentation. The nps2nps6ftrl triple mutants were unable to grow on MM, and while iron supplementation slightly restored growth, it did not do so to the extent that it restored growth of nps6ftrl mutants (Fig. 2). These data demonstrate that, while RIA is dispensable for growth and development in the presence of NPS6, it functions as a backup mechanism in $n p s 6$ mutants.

To determine if iron mutants are better able to utilize one form of iron over another, we tested growth on CMX with various concentrations of chelated and unchelated ferric and ferrous iron (Supplementary Fig. S2). There was no difference among iron sources used for supplementation; a threshold concentration between 2.5 and $25 \mu \mathrm{M}$ of ferric or ferrous salt (ferric chloride and ferrous sulfide, respectively) as well as chelated ferric iron (ferric citrate) was able to supplement growth. 
Examination of nps6ftrl and nps2nps6ftrl in CM liquid cultures revealed that hyphal growth is abnormal without supplemental iron (Supplementary Fig. S3). The nps6ftrl cultures grew poorly and with an abnormal wavy morphology, while nps2nps6ftrl growth was extremely limited and cultures contained senescent melanized hyphae. Supplemental iron alleviated the wavy growth defect of nps6ftrl cultures, but abundance of hyphae was still reduced when compared with WT. The nps2nps6ftrl cultures also improved in growth with supplemental iron but with defective wavy morphology comparable to that seen in nps6ftrl cultures without iron.

\section{nps2nps6 and nps2nps6ftr1 strains are affected in sexual development.}

C. heterostrophus nps 2 strains fail to form asci and ascospores in homozygous nps 2 crosses on MM, although the number of pseudothecia formed per square centimeter of leaf surface and the morphology of these pseudothecia are like WT crosses (Oide et al. 2007). The nps6 strains are not affected in sexual development (Oide et al. 2007). C. heterostrophus nps 2 nps6 strains, when crossed to a WT strain of opposite mating type, showed WT numbers of pseudothecia and WT fertility (Fig. 3A and C). No significant reduction in the number of pseudothecia per square centimeter of leaf area or in the number of asci per pseudothecium was observed as compared with WT crosses, whether nps2nps6 strains acted as male or female (Supplementary Fig. S4). Furthermore, regardless of mating type of the nps2nps6 strains, crosses between WT and $n p s 2 n p s 6$ strains were as fertile as WT $\times$ WT crosses. Homozygous nps $2 n p s 6$ crosses did not form pseudothecia (Fig. 3C and D), in contrast to homozygous nps 2 crosses, which form pseudothecia with WT morphology but no asci (Fig. 3E and F) (Oide et al. 2007).

Increasing concentrations of iron partially restored ability of the nps2nps6 strains to develop pseudothecia (Fig. 3E), indicating that the defect in pseudothecium development of $n p s 2 n p s 6$ strains is at least partially due to iron starvation. Asci and ascospores were observed in these pseudothecia, although the number per pseudothecium was much less than that of WT crosses and homozygous nps 2 mutant crosses supplemented with iron (Fig. 3F). Homozygous nps2ftrl crosses were like $n p s 2 \times n p s 2$ crosses, producing barren pseudothecia, while homozygous ftrl $\times f t r l$ and $n p s 6 f t r l \times n p s 6 f t r l$ crosses were like WT crosses (data not shown). RIA, therefore, has no obvious

Table 1. Cochliobolus heterostrophus strains used in this study.

\begin{tabular}{|c|c|c|}
\hline Strain $^{a}$ & Genotype $^{\text {b }}$ & Comments and References \\
\hline C4 (ATCC 48331) & Tox1;MAT1-2 & Race T, inbred laboratory strain, wild type (WT); Leach et al. 1982 \\
\hline $\mathrm{C} 2$ & Tox1;MAT1-1;alb1 & As above \\
\hline C9 & Tox $1 ; M A T 1-1$ & As above \\
\hline CB11 & Tox1;MAT1-2;alb1 & Race T, inbred laboratory strain, WT (Bronson 1988) \\
\hline ChDsred & $R F P ;$ hyg $B ; M A T 1-2$ & Dsred (Matz et al. 1999) expressed under the citrate lyase promoter in pIGREDPAPA \\
\hline Chnps2-1 & nps2;hygB;MAT1-2 & Oide et al. 2007 \\
\hline Chnps2-2 & nps $2 ;$ hyg B;MAT1-2 & As above \\
\hline Ch1449-T1-1 & nps $2 ;$ hyg B; MAT1-2 & As above \\
\hline Ch1449-T1-5 & nps 2;hygB;MAT1-1;alb1 & As above \\
\hline Chnps6-1 & nps6; hygB;MAT1-2 & Oide et al. 2006 \\
\hline Chnps6-1R2 & nps6;hygB;MAT1-1 & As above \\
\hline Chftr1-1 & ftrl; hygB;MAT1-2 & This study, iron permease FTR 1 deletion \\
\hline Chftr1-2 & ftr1; hyg B;MAT1-2 & As above \\
\hline Ch1721-R1 & ftr1;hygB;MAT1-1;alb1 & This study, progeny of cross Chftr $1-1 \times \mathrm{C} 2$ \\
\hline Ch1721-R3 & ftr1;hygB;MAT1-2;alb1 & As above \\
\hline Chfet3-1 & fet $3 ;$ hygB $;$ MAT1-2 & This study, FET3 deletion \\
\hline Chfet3-2 & fet $3 ;$ hyg $B ;$ MAT1-2 & As above \\
\hline Chfet3ftr1-1 & fet $3 ; f \operatorname{tr} 1 ;$ hyg $B ; M A T 1-2$ & This study, deletion of both FET3 and FTRI \\
\hline Chfet3ftr1-2 & fet3;ftr1;hygB;MAT1-2 & As above \\
\hline Ch1495-T1-1 & nps $2 ; n p s 6 ;$ hygB; $B A T 1-2$ & This study, progeny of Chnps2-1 $\times$ Chnps6-1R2 \\
\hline Ch1495-T1-6 & nps $2 ;$ nps6;hygB;MAT1-1 & As above \\
\hline Ch1495-T1-8 & nps $2 ;$ nps6; hygB; $B A T 1-2$ & As above \\
\hline Ch1496-T1-1 & nps2;nps6;hygB;MAT1-1 & This study, progeny of Chnps2-2 $\times$ Chnps6-1R2 \\
\hline Ch1496-T1-8 & nps $2 ;$ nps $6 ;$ hyg $B ; M A T 1-1$ & As above \\
\hline Ch1722-R1 & nps6;ftrl;hygB;MAT1-1 & This study, progeny of Chnps6-1R $2 \times$ Chftr1-1 \\
\hline Ch1722-R3 & nps6;ftr1;hygB;MAT1-2 & As above \\
\hline Ch1722-R4 & nps6;ftrl;hygB;MAT1-2 & As above \\
\hline Ch1723-T2-5 & nps $2 ; f t r 1 ;$ hyg $B ; M A T 1-2$ & This study, progeny of Chftr1-1 and 1449-T1-5 \\
\hline Ch1731-T6-3 & nps $2 ;$ nps $6 ;$ tr $1 ;$ hyg $B ; M A T 1-1$ & This study, progeny of Ch1722-R3 $\times$ Ch1449-T1-5 \\
\hline Ch1743-T6-1 & nps2;nps6;ftr1;hygB;MAT1-2 & This study, progeny of Ch1721-R3 $\times$ Ch1495-T1-6 \\
\hline Ch1744-T6-6 & nps $2 ; n p s 6 ; f t r 1 ;$ hygB, alb1 & Repeat of above cross \\
\hline Ch1754-R6 & nps6;fet $3 ;$ hyg B & This study, progeny of Chnps6-1R $2 \times$ Chfet $3-1$ \\
\hline Ch1754-R7 & nps6;fet3; hyg B & As above \\
\hline Ch1755-R11 & nps6;fet $3 ;$ ftr $1 ;$ hyg $B$ & This study, progeny of Chnps6-1R $2 \times$ Chfet $3 \mathrm{ftr} 1$ \\
\hline Ch1755-R18 & nps6:fet3;ftr $1 ;$ hyg $B$ & As above \\
\hline Ch1761-R7 & RFP; hygB;MAT1-1 & This study, progeny of Dsred $\times \mathrm{C} 2$ \\
\hline Ch1761-R9 & RFP;hygB;MAT1-1 & As above \\
\hline Ch1762-T1-1 & $R F P ; n p s 6 ; h y g B$ & This study, progeny of Chnps6-1 $\times$ Ch1761-R-7 \\
\hline Ch1764-T1-1 & RFP;nps6;ftrl; hygB & This study, progeny of $1722-\mathrm{R}-3 \times \mathrm{Ch} 1761-\mathrm{R}-7$ \\
\hline Ch1765-T2-3 & RFP;nps $2 ; n p s 6 ;$ hygB & This study, progeny of $1495-\mathrm{T} 1-1 \times$ Ch1761-R-7 \\
\hline Ch1765-T3-5 & RFP; nps $2 ;$ nps6; hygB & As above \\
\hline Ch1766-R2 & RFP;nps2;nps6;ftr1;hygB & This study, progeny of Ch1743-T6-1 × Ch1761-R-7 \\
\hline Ch1768-T1-7 & nps $2 ;$ ftr $1 ;$ hyg $B ; M A T 1-1$ & This study, progeny of Ch1723-T2-5 × CB11 \\
\hline Ch1768-T5-7 & nps2;ftr1;hygB;MAT1-1 & As above \\
\hline
\end{tabular}

${ }^{\text {a }}$ Chnps2-1 is a strain in which NPS2 is deleted, '-1' indicates transformant number 1; 1449-T1-1 is cross number 1449; T1-1 is tetrad number 1, ascospore 1. 1766-R2 is the second random progeny recovered (rather than part of a tetrad) from cross number 1766.

${ }^{\mathrm{b}}$ Strains are pigmented unless denoted albl in genotype. If mating type is not included, it was not tested and is unknown. 
role in sexual development, whereas the NPS6 siderophore has a role secondary to NPS2 siderophore. This suggests that the phenotype observed in nps2nps6 mutants compared with $n p s 2$ mutants is not related to the role of coprogen in iron acquisition (because nps6ftrl mutants, which are totally impaired in iron acquisition, display WT sexual development) but, instead, to its contribution to intracellular iron or to ROS status.

\section{nps6ftr1 and nps2nps6ftr1 strains are affected in asexual development.}

Asexual spore (conidium) production was assessed on CMX and MM with and without supplemental iron for all strains (Fig. 4). On CMX, WT, nps2, nps6, ftrl, nps $2 f t r 1$, and nps $2 n p s 6$ strains all produced $1 \times 10^{6}$ to $7 \times 10^{6}$ conidia per plate with or without $150 \mu \mathrm{M}$ supplemental ferric citrate. The nps6ftrl, nps6fet3, and nps6fet3ftrl strains, however, produced approximately 50-fold fewer conidia than this on CMX; supplementation with ferric citrate restored conidium production to WT levels. The nps2nps6ftrl strains failed to produce conidia on CMX, but conidium production could be restored with supplemental iron, albeit to 75-fold fewer conidia per plate than WT. WT and strains $n p s 2$, ftrl, and $n p s 2 f t r 1$ produced similar numbers of conidia on MM as they did on CMX, while nps6 and nps $2 n p s 6$ strains produced 20- to 40-fold fewer than WT. The nps6ftr1, nps6fet 3 , nps6ftrlfet3, and nps2nps6ftrl strains did not produce conidia on MM with or without supplemental iron. High-affinity iron acquisition is required, therefore, for conidial development.

\section{Iron acquisition mutants can be fed iron by other strains.}

High-performance liquid chromatography (HPLC) analyses revealed that neither intracellular (ferricrocin, synthesized by

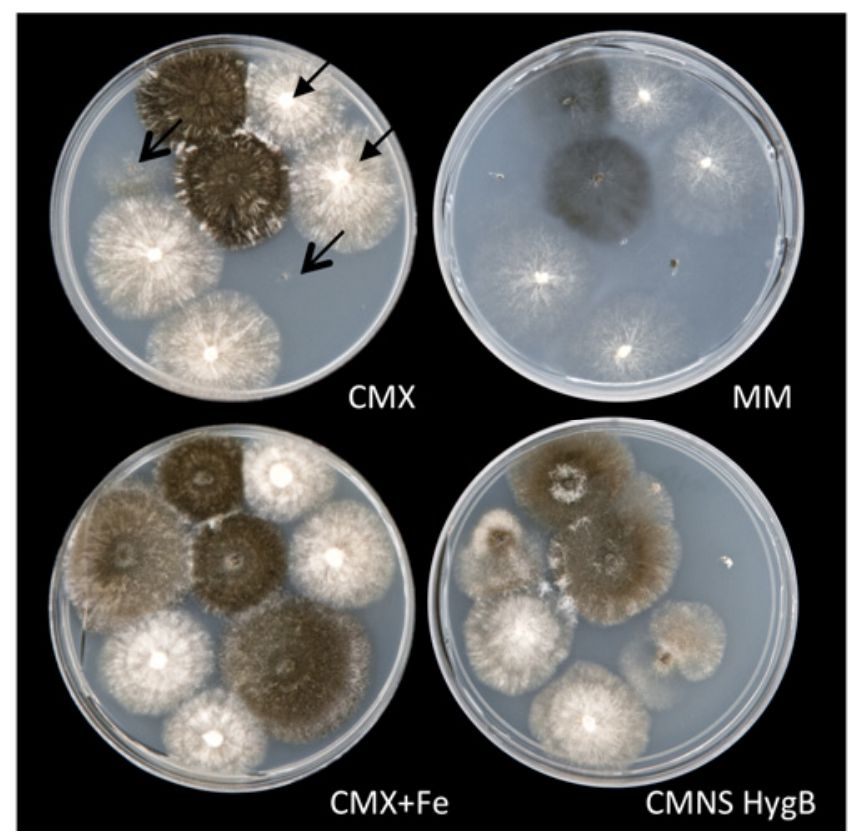

Fig. 1. Identification of triple mutants by plating tetrads on differential media. Eight ascospores from a single ascus isolated from a cross between nps6ftr1 (strain Ch1722-R3) and nps2 (albino strain Ch1449-T1-5) mutants were plated on complete medium with xylose (CMX), minimal medium (MM), CMX + iron, and complete medium without salt solution (CMNS) + hygromycin B. Position of strains is the same in each plate. Wild-type (WT) progeny are indicated with closed arrows, and triple nps $2 n p s 6 f t r 1$ mutant progeny with open arrows. All eight progeny grow on CMX + iron. The nps2nps6ftrl progeny do not grow on CMX and MM, indicating that they require iron. WT progeny do not grow on medium containing hygromycin $\mathrm{B}$. The ALB1 gene controlling pigmentation segregates $1: 1$ and serves as a control for single gene segregation.
Nps2 and found in mycelium) nor extracellular (coprogen, synthesized by Nps6 and secreted into the broth) siderophores were detectable in the nps2nps6 sample while both were detected in WT (Fig. 5). As reported earlier (Oide et al. 2006, 2007), no ferricrocin was detected in the nps 2 sample, although coprogens were detected. In contrast, no coprogens were detected in the nps 6 sample, although a small amount of ferricrocin was detected in the mycelial fraction (Fig. 5). Quantitative analyses of siderophore biosynthesis (Table 3 ) revealed that the nps 2 mutant produced two to six times more coprogens (milligrams per liter of broth) compared with WT, while the nps6 mutant produced approximately twice as much ferricrocin (milligrams per gram of mycelium) as WT did, suggesting that the $\mathrm{N}^{5}$-hydroxyornithine precursor shared by the two pathways is diverted to producing more ferricrocin when Nps6 is not present to synthesize coprogen.

When plated alone, nps2nps6ftrl is incapable of growing on CMX (without iron supplement). When plated alongside other strains, such as when tetrad progeny are plated together (Fig. 1), these strains were able to grow, albeit in abnormal patterns. Noticing this, we developed an assay to test whether this phenomenon was due to the ability of the nps2nps6ftrl strain to utilize compounds (such as siderophores) produced by other strains. Mycelia and conidia of nps2nps6ftrl were applied to complete medium without salt solution (CMNS) plates with hygromycin B at varying distances from hygromycin-sensitive WT mycelia and conidia (Fig. 6). After 2 days, nps 2 nps6ftr 1 mutants closer to the WT strain were able to grow but not those plated farther away from the WT strain. Furthermore, colony growth habit was not radial; mutants grew towards the

Table 2. Crosses performed in this study

\begin{tabular}{|c|c|}
\hline Cross & Parent strains $^{a}$ \\
\hline Wild type $(\mathrm{WT}) \times \mathrm{WT}$ & $\mathrm{C} 4 \times \mathrm{C} 2$ \\
\hline $\mathrm{WT} \times \mathrm{WT}$ & $\mathrm{C} 4 \times \mathrm{C} 9$ \\
\hline$f t r l \times W T$ & Chftr1-1 $\times \mathrm{C} 2$ \\
\hline$f t r 1 \times n p s 6$ & Chtr1-1 × Chnps6-1R2 \\
\hline$f t r 1 \times n p s 2$ & Chftr1-1 × Ch1449-T1-5 \\
\hline fet $3 \times \mathrm{WT}$ & Chfet $3-1 \times \mathrm{C} 2$ \\
\hline$n p s 2 \times n p s 6$ & Chnps2-1 $\times$ Chnps6-1R2 \\
\hline$n p s 2 \times n p s 6$ & Chnps2-1 $\times$ Chnps6-1R2 \\
\hline ftrlnps $6 \times n p s 2$ & Ch1722-R-1 × Chnps2-1 \\
\hline$f t r 1 \times n p s 2 n p s 6$ & Ch1721-R-3 × Ch1495-T1-6 \\
\hline fet $3 \times n p s 6$ & Chfet3-1 $\times$ Chnps6-1R2 \\
\hline fet $3 f t r 1 \times n p s 6$ & Chfet3ftr1-1 × Chnps6-1R2 \\
\hline Dsred $\times$ WT & ChDsred $\times \mathrm{C} 2$ \\
\hline Dsred $\times n p s 6$ & Chnps6-1 × Ch1761-R7 \\
\hline Dsred $\times n p s 2 n p s 6$ & Ch1495-T1-1 × Ch1761-R7 \\
\hline Dsred $\times n p s 6 f t r 1$ & Ch1722-R3 × Ch1761-R7 \\
\hline Dsred $\times$ nps 2 nps $6 f t r 1$ & Ch1743-T6-1 × Ch1761-R7 \\
\hline$f t r 1 \times f t r l$ & Chftr1-1 × Ch1721-R2 \\
\hline$n p s 2 \times n p s 2$ & Ch1499-T1-1 × Ch1449-T1-5 \\
\hline$n p s 2 n p s 6 \times \mathrm{WT}$ & Ch1495-T1-1 × C2 \\
\hline nps 2 nps $6 \times \mathrm{WT}$ & Ch1495-T1-8 $\times$ C2 \\
\hline nps $2 n p s 6 \times \mathrm{WT}$ & Ch1496-T1-1 × C4 \\
\hline nps $2 n p s 6 \times \mathrm{WT}$ & Ch1496-T1-8 × C4 \\
\hline nps 2 nps $6 \times n p s 2 n p s 6$ & Ch1495-T1-1 × Ch1496-T1-1 \\
\hline nps 2 nps $6 \times n p s 2 n p s 6$ & Ch1495-T1-1 × Ch1496-T1-8 \\
\hline nps 2 nps $6 \times n p s 2 n p s 6$ & Ch1495-T1-8 × Ch1496-T1-1 \\
\hline nps $2 n p s 6 \times n p s 2 n p s 6$ & Ch1495-T1-8 × Ch1496-T1-8 \\
\hline nps $6 f t r 1 \times n p s 6 f t r 1$ & Ch1722-R1 × Ch1722-R3 \\
\hline nps $6 f t r 1 \times n p s 6 f t r 1$ & Ch1722-R1 × Ch1722-R4 \\
\hline$n p s 6 f t r 1 \times \mathrm{WT}$ & Ch1722-R1 × C4 \\
\hline$n p s 2 f t r 1 \times n p s 2 f t r 1$ & Ch1723-T2-5 × Ch1768-T1-7 \\
\hline$n p s 2 f t r 1 \times n p s 2 f t r 1$ & Ch1723-T2-5 × Ch1768-T5-7 \\
\hline$n p s 2 f t r 1 \times \mathrm{WT}$ & Ch1723-T2-5 × C9 \\
\hline
\end{tabular}

${ }^{a}$ Chnps2-1 is a strain in which NPS2 is deleted, '-1' indicates transformant number 1 ; 1449-T1-1 is cross number 1449; T1-1 is tetrad number 1 , ascospore $1.1766-\mathrm{R} 2$ is the second random progeny recovered (rather than part of a tetrad) from cross number 1766. 
WT strain more vigorously than they did in the opposite direction. The nps2nps6ftrl strain is, therefore, able to utilize one or more factors biosynthesized by WT, if close by, and to grow in the direction of the supplier.
To investigate further, plates were set up with culture filtrates from WT, nps6, nps 2 , and nps 2 nps 6 strains placed in wells in the agar at the four corners of the experiment (Fig. 6). The nps2nps6ftrl scrapings that were adjacent to WT filtrate were
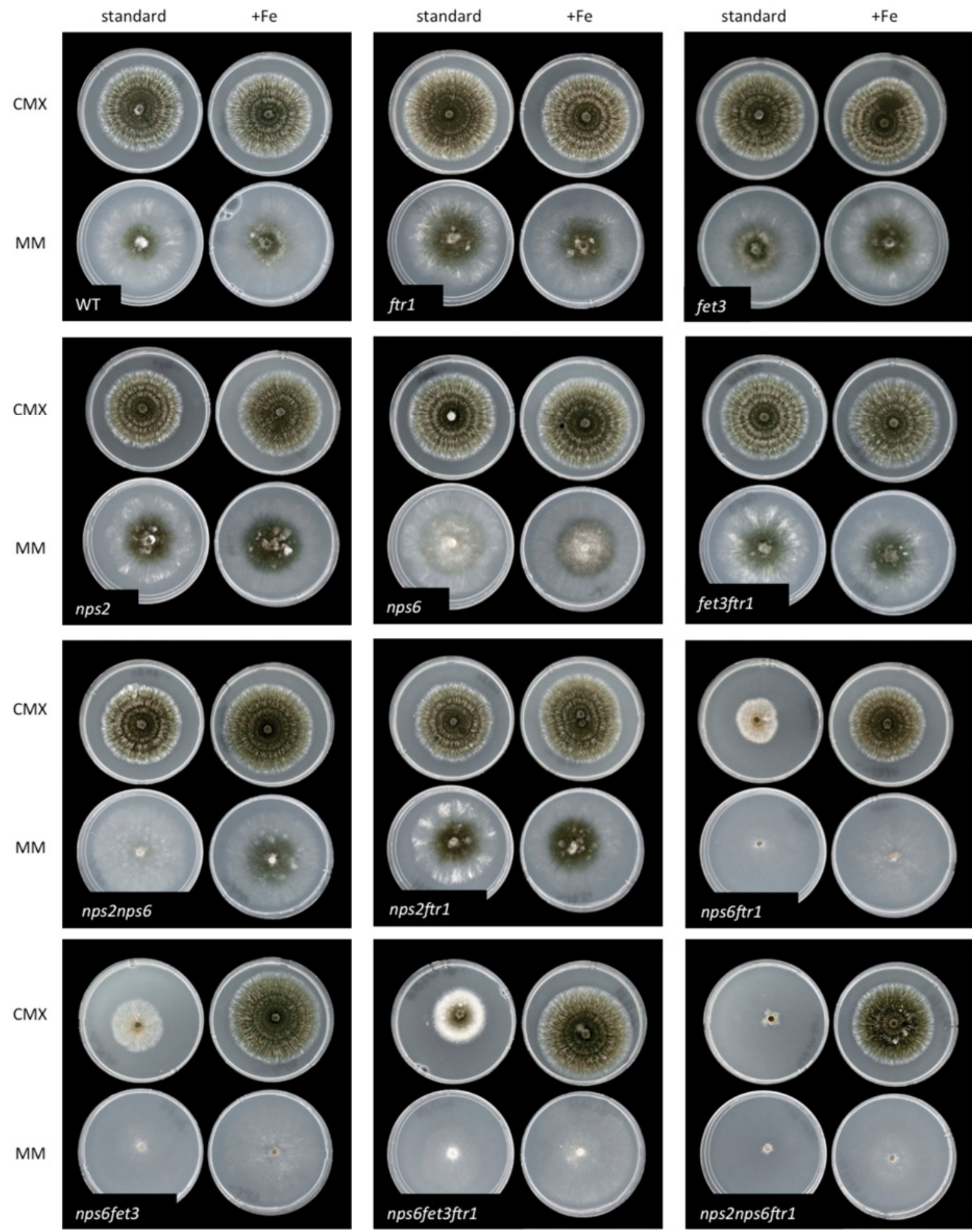

Fig. 2. Double and triple iron acquisition mutants are impaired in growth on complete medium with xylose (CMX) and minimal media (MM). The ftrl (strain Chftr1-1), fet3 (strain Chfet3-1), and fet3ftr1 (strain Chfet3ftr1-1) reductive iron assimilation (RIA) mutants and the $n p s 2$ (strain Chnps2-1), nps6 (strain Chnps6-1), and nps2nps6 (strain Ch1495-T1-1) siderophore mutants grow like wild type (WT) (strain C4) on CMX and MM and do not benefit from supplemental iron. Combined RIA and extracellular siderophore mutants nps6ftr1 (strain Ch1722-R1), nps6fet3 (strain Ch1754-R6), and nps6fet3ftr1 (strain Ch1755-R11), however, are reduced in growth and pigmentation on both CMX and MM. Adding supplemental iron (100 $\mu$ M ferric citrate) to CMX restores colony growth, although it remains sparse on MM. The nps2nps6ftrl (strain Ch1731-T6-3) triple mutants are extremely reduced in growth on CMX, extending only a few millimeters past the inoculum plug. Supplemental iron restores growth to resemble that of nps6ftrl. The nps2nps6ftrl mutants are completely unable to grow on MM without supplemental iron, which only partially supplements growth. 
well-restored in growth. Those next to $n p s 2$ filtrate also grew, however, nps2nps6ftrl scrapings adjacent to nps6 and nps2nps6 filtrates were unable to grow. We hypothesize that coprogen siderophores biosynthesized by Nps6 in WT and nps 2 mutants diffuse in the medium and can be used by the nps 2 nps6ftrl mutant to complement its growth defect. Both nps6 and nps2nps6 strains lack coprogens (Fig. 5) and, therefore, cannot feed nps2nps6ftrl strains.

\section{Iron acquisition mutants are sensitive} to oxidative and iron stress.

Deletion of NPS6 leads to hypersensitivity to oxidative stress and iron depletion in C. heterostrophus (Oide et al. 2006). De- letion of NPS2, however, does not affect sensitivity to these stresses (Oide et al. 2007). The RIA and combinatorial mutants generated in this study were assayed for sensitivity to oxidative stress, using hydrogen peroxide $\left(\mathrm{H}_{2} \mathrm{O}_{2}\right)$ and the superoxide generator $\mathrm{KO}_{2}$, and also to iron stress, using the membranepermeable iron chelator 2,2-dipyridyl (2DP) and the membraneimpermeable iron chelator bathophenanthroline disulfonate (BPS) (Fig. 7). The ftrl mutants grew like WT at each stressor concentration tested.

As previously described (Oide et al. 2006), nps6 mutant growth was more inhibited than WT for each stress condition, when stress concentrations allowed a distinction (Fig. 7). The nps 2 ftrl sensitivity largely mirrored that of WT and ftrl mu-
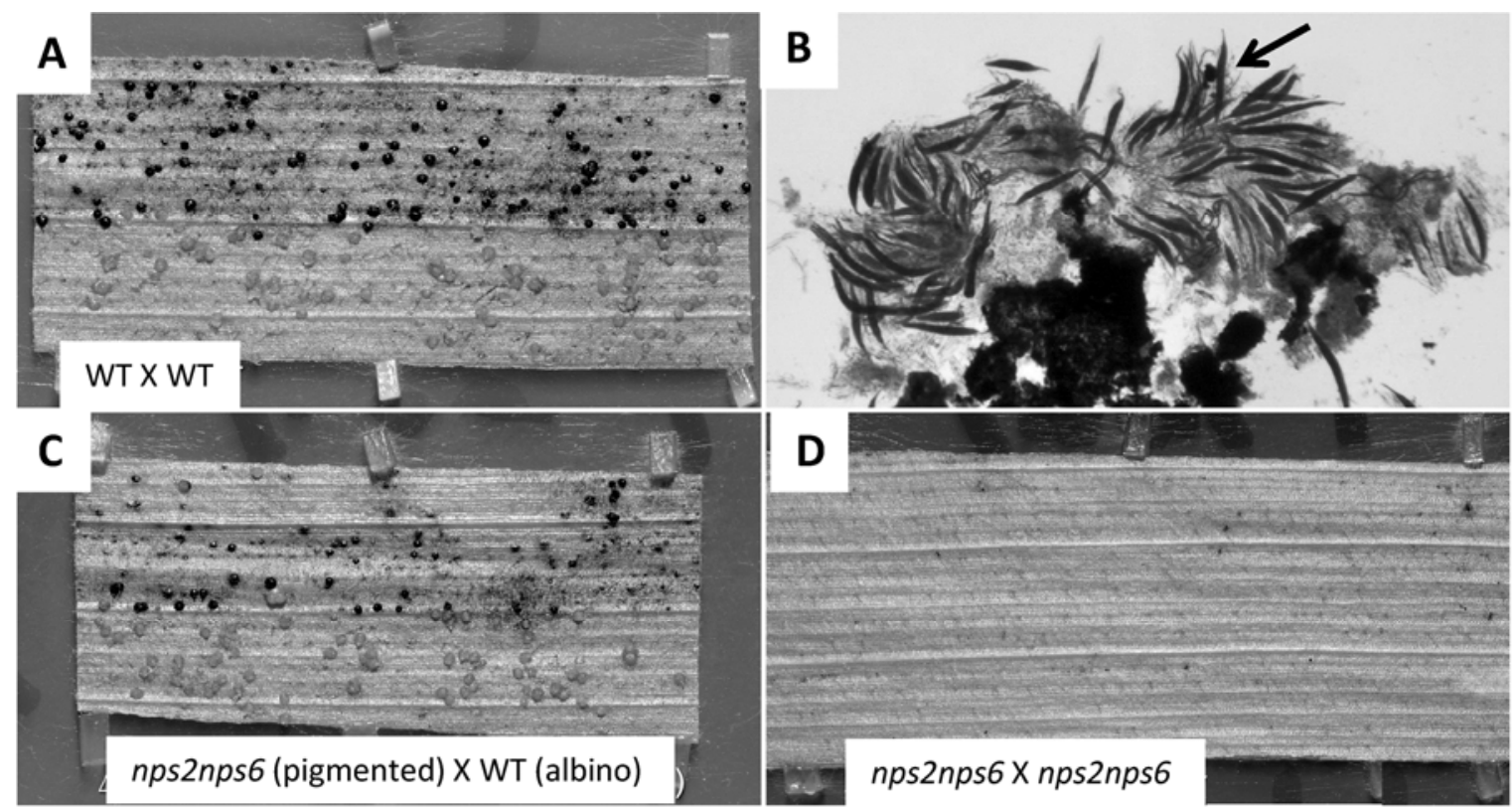

E

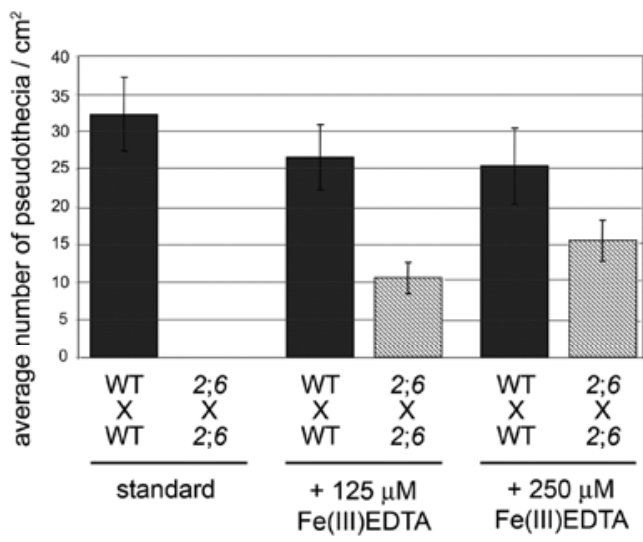

$\mathbf{F}$

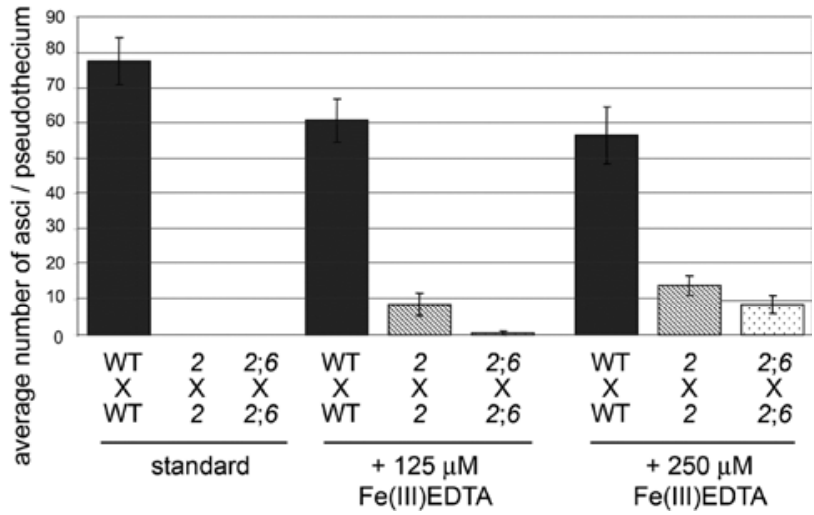

Fig. 3. Without supplemental iron, nps2nps6 strains fail to develop pseudothecia in homozygous crosses. A, Corn leaf substrate bearing pseudothecia, 21 days after crossing wild type (WT) pigmented strain C4 and WT albino strain C2. Both pigmented and albino pseudothecia form, indicating that each parent is hermaphroditic. B, Contents of a crushed pseudothecium from a WT $\times$ WT cross, demonstrating typical fertility (many asci [arrow] and ascospores). C, Corn leaf substrate bearing pseudothecia 21 days after crossing nps2nps6 $\times$ WT strains (Ch1495-T1-1 X C2). When crossed to a WT strain, nps2nps6 strains develop pseudothecia as well as WT strains do. D, In homozygous nps2nps6 crosses (strain Ch1495-T1-1 × Ch1496-T1-1), nps2nps6 strains fail to develop pseudothecia. E, The average number of pseudothecia per square centimeter of leaf is shown for homozygous WT and nps $2 n p s 6$ crosses. Ten replicates were set up for each cross and for each condition. Application of ferric EDTA restores ability to develop pseudothecia to the nps2nps6 strains in homozygous $n p s 2 n p s 6$ crosses (abbreviated $2 ; 6 \times 2 ; 6$ ), although the number of pseudothecia formed is less than homozygous WT crosses. A reduction in the number of pseudothecia per square centimeter of corn leaf was observed in WT crosses when iron was applied, implying an adverse effect of iron overload. F, The average number of asci per pseudothecium is shown for homozygous WT, nps2, or nps2nps6 crosses. Twenty pseudothecia were opened for each cross and for each condition. Error bars indicate $95 \%$ confidence intervals. Application of iron restores ability to develop asci and ascospores to the $n p s 2$ and $n p s 2 n p s 6$ strains (abbreviated $2 \times 2$ and $2 ; 6 \times 2 ; 6$ ), although the number of asci per pseudothecium is smaller compared with that of WT crosses. The number of asci per pseudothecium developed by homozygous nps $2 n p s 6$ crosses is smaller than that of homozygous $n p s 2$ crosses, further demonstrating that iron metabolism during sexual development is more severely affected in the $n p s 2 n p s 6$ strains than in the $n p s 2$ strains. 
tants, suggesting lack of intracellular siderophores, and the RIA mechanism does not impact resistance to these stresses. Although nps6ftrl mutant growth was delayed on MM without stress, growth was comparable to that of the nps6 strain on $\mathrm{H}_{2} \mathrm{O}_{2}$ and $\mathrm{KO}_{2}$. Growth of nps2nps6 was more sensitive to oxidative stress than nps6 and nps6ftrl strains, when stress concentrations allowed a distinction. The nps2nps6ftrl strain was not included, as it is not capable of growing on MM. Deletion of NPS2, therefore, affects oxidative stress only in the absence of NPS6, suggesting that intracellular siderophores do play a role in this process. RIA, on the other hand, does not play a clear role in oxidative stress sensitivity, even in the absence of NPS6, since nps6ftrl strains showed the same sensitivity as nps6 strains (Fig. 7; Supplementary Fig. S5).

Low concentrations $(75 \mu \mathrm{M})$ of 2DP did not significantly impair the growth of any strain and, in some cases, growth was greater than on MM. At high concentrations $(150 \mu \mathrm{M})$ of $2 \mathrm{DP}$, WT, ftrl, and nps2ftrl strains were able to grow, but nps6, nps2nps6, and nps6ftrl strains were not. Growth of WT, ftrl, and nps 2 ftrl strains at the highest concentrations $(300 \mu \mathrm{M})$ of BPS was comparable to that of the corresponding strain's growth on MM, suggesting they were not affected by this stressor. The nps6, nps2nps6, and nps6ftrl strains, on the other hand, were significantly reduced in growth at all concentrations tested as compared with WT, but double mutants were not consistently more sensitive than nps6 single mutants (Fig. 7; Supplementary Fig. S6).

\section{Iron acquisition and iron storage mutants are affected in virulence.}

It was determined previously that deletion of $C$. heterostrophus NPS6 leads to reduction in virulence to maize, while deletion of NPS 2 does not affect virulence (Lee et al. 2005; Oide et al. 2006). Virulence assays done in the current study confirmed the reduced virulence of the nps 6 mutant but indicated that ftrl and nps2ftrl mutants are not reduced in virulence compared with WT (Fig. 8A). The nps2nps6 and nps6ftrl strains caused further reductions in virulence compared with nps6 strains; nps2nps6ftrl mutants were extremely reduced in virulence, forming atypical small white lesions rather than expanding lesions in various stages of necrosis. This reduction in virulence is not due to morphological defects of the nps 2 nps6ftrl conidia, as they germinated as well as WT conidia when spore suspensions were placed on glass slides (Supplementary Fig. S7). Adding either exogenous iron (Fig. 8B) or the antioxidant ascorbic acid (data not shown) or both to the inoculum and leaf surface did not significantly increase average lesion size for any strain used. The average lesion size of the nps2nps6ftrl mutant was slightly (but not significantly) complemented by exogenous iron (Fig. 8A). The presence of NPS6 is, therefore, of primary importance for virulence, but the absence of NPS 2 and FTR 1 can each affect virulence in the absence of NPS6.

A key goal of this study was to differentiate between the roles iron and oxidative stress play in the virulence phenotypes of iron acquisition mutants. To this end, we applied the ROSstaining dye 3,3'-diaminobenzidine (DAB) to infected leaves. At $24 \mathrm{~h}$, DAB accumulation (Fig. 9, arrow) could be seen in WT- and nps6-infected leaves, presumably immediately surrounding points of infection, indicating the presence of ROS. Leaves challenged with nps6ftrl and nps2nps6 mutants had smaller, more discrete spots of DAB accumulation, and those challenged with the nps2nps6ftrl mutant looked more like uninoculated controls, lacking DAB accumulation. At 5 days after infection, when lesions are plainly visible on the leaf, DAB staining was profuse in WT- and nps6-mutant challenged leaves. Leaves challenged with nps6ftrl and nps2nps6 mutants had discrete lesion-shaped DAB stains, often with stain accumulating at the edge without staining in the middle. The nps2nps6ftrl-inoculated and uninoculated leaves did not show DAB accumulation. The tiny lesions on leaves (Fig. 8) and the lack of DAB staining (Fig. 9) suggest that nps2nps6ftrl mutants elicit a different response from the plant than all other strains used. Loss of DAB staining in nps2nps6, nps6ftrl, and nps2nps6ftrl infections suggests iron acquisition mutants may be deficient in producing virulence-promoting ROS. Alternatively, DAB accumulation may be a product of host cell death and iron mutants are less able to kill host tissue, resulting in less DAB accumulation.

Iron-acquisition mutants have impaired in planta growth.

To further investigate how virulence is impaired in these mutants, Dsred (red fluorescent protein)-expressing strains were observed in planta at $24 \mathrm{~h}$ and 5 days after inoculation. At $24 \mathrm{~h}$, no clear difference in spore germination or appressorium formation was observed between WT and the nps6, nps6ftrl, or nps2nps 6 mutants (not shown). The extent of colonization by both nps6ftrl and nps2nps6 mutants at this time, however, was reduced compared with WT or nps6. For all strains, sites of fungal entry were often accompanied by autofluorescence originating from the challenged epidermal cell. After 5 days, nps6ftr 1 and nps $2 n p s 6$ mutant growth had halted after spreading 100 to $500 \mu \mathrm{m}$, whereas comparable regions were extensively colonized by WT and nps6 strains (Fig. 10). Combined with lesion measurements (Fig. 8B) and DAB staining (Fig. 9) data, this suggests that both nps2nps6 and nps6ftrl mutants are able to enter the leaf and initiate infection, but they fail to colonize extensively. Patches of accompanying autofluorescence likely correspond to host defense response (Fig. 10, bottom row).

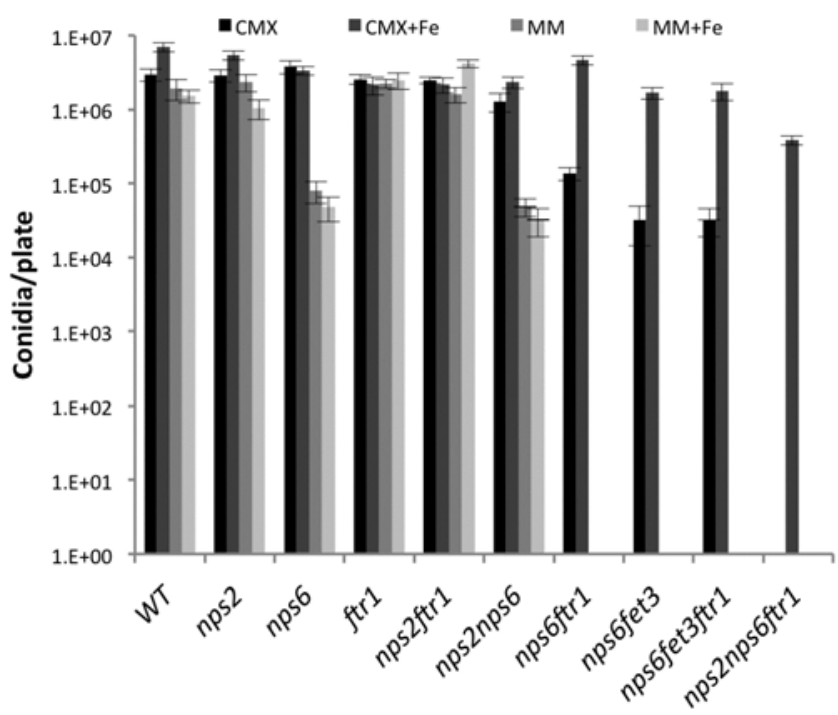

Fig. 4. The nps6ftrl and nps2nps6ftrl mutants are impaired in asexual spore production. On complete medium with xylose (CMX), wild type (WT) (strain C4), nps2 (strain Chnps2-1), nps6 (strain Chnps6-1), ftr1 (strain Chftr1-1), nps2ftrl(strain Ch1723-T2-5), and nps2nps6 (strain Ch1495-T11) mutants all produced between $1,280,000$ to $6,960,000$ conidia per plate with or without supplemental iron. While nps6ftrl (strain Ch1722-R1), nps6fet3 (strain Ch1754-R6), and nps6fet3ftrl (strain Ch1755-R11) mutants produced between 32,000 and 136,000 conidia (50- to 100-fold fewer). nps2nps6ftrl mutants did not produce conidia on CMX. With $50 \mu \mathrm{M}$ supplemental iron, nps6ftrl, nps6fet3, and nps6fet $3 f t r 1$ conidia production was restored to WT levels. The nps2nps6ftrl mutants were also able to produce conidia (but 10-fold fewer than WT) with supplemental iron. On minimal medium (MM), conidia production was reduced 20- to 60-fold for both $n p s 6$ and nps $2 n p s 6$ compared with WT, with or without supplemental iron. The nps6ftr1, nps6fet 3 , nps6fet3ftr1, and nps2nps6ftr1 mutants did not produce conidia on MM with or without supplemental iron. 
broth
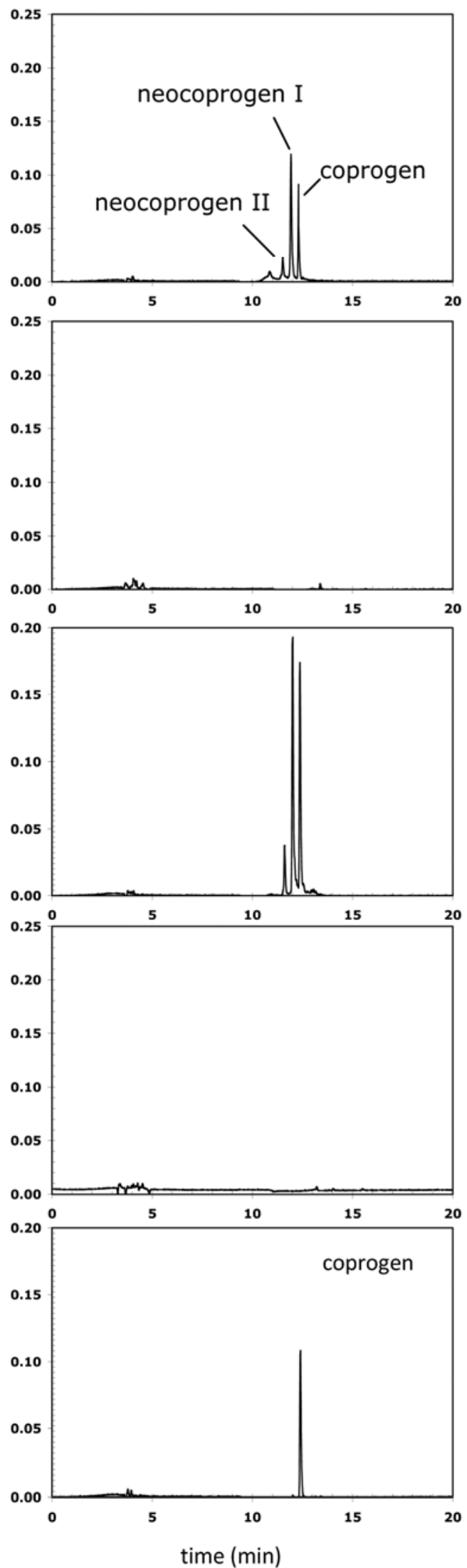

mycelium
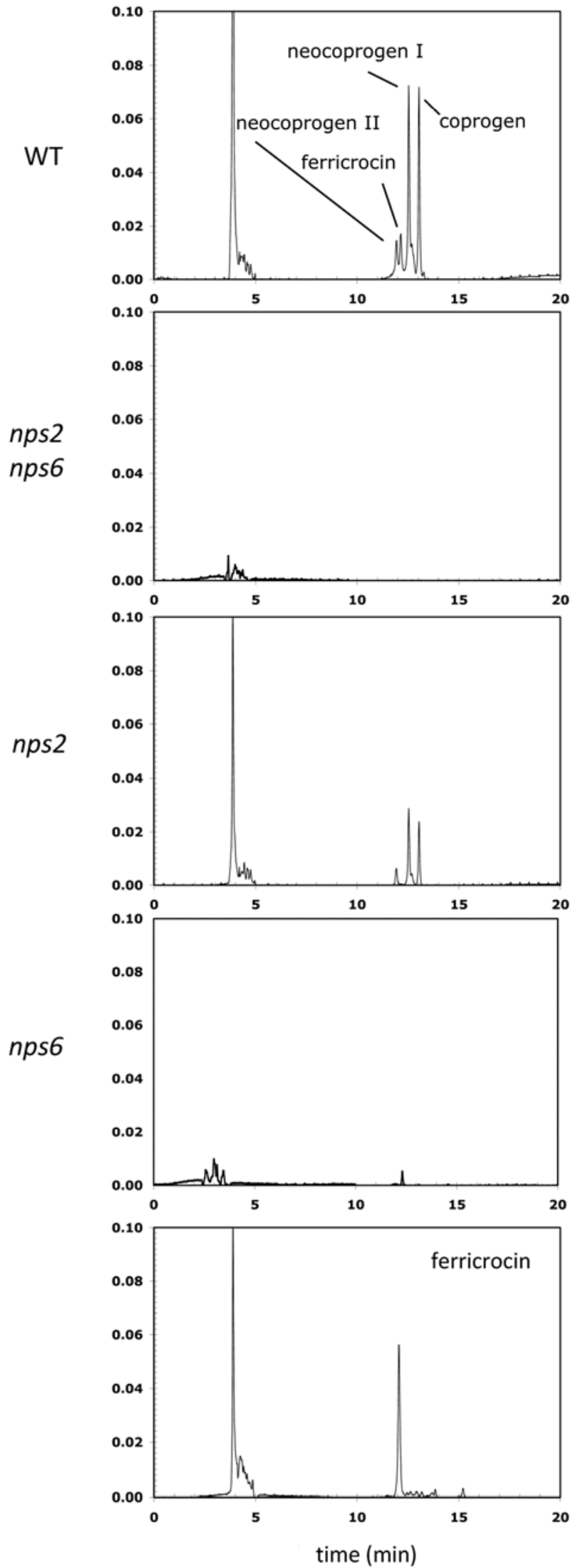

Fig. 5. The nps2nps6 mutants do not produce siderophores. High-performance liquid chromatography analyses of wild-type (WT) (strain C4), $n p s 2 n p s 6$ (strain Ch1495-T1-1), nps2 (strain Chnps2-1), and nps6 (strain Chnps6-1) strains. Samples were extracted from broth (left) and mycelium (right) of fungal cultures grown under iron-depleted conditions. Coprogen and ferricrocin standards are shown in the bottom panel. WT produces coprogens and ferricrocin as extra- and intracellular siderophores, respectively. In contrast, no coprogens or ferricrocin were detected in the nps $2 n p s 6$ sample, demonstrating that double deletion of NPS2 and NPS6 completely abolished siderophore biosynthesis. 
For the nps2nps6ftrl triple mutant, there were fewer conidia to sample at both $24 \mathrm{~h}$ and 5 days. Those that could be observed were able to germinate and form appressoria (Fig. 10), as seen in vitro. No in planta fungal growth beyond appressorium formation and the initial site of penetration could be observed at $24 \mathrm{~h}$ or 5 days. Combined with the atypical tiny lesions observed on whole leaves (Fig. 8) and the lack of DAB staining (Fig. 9), this suggests that nps2nps6ftrl does not advance as a pathogen beyond appressorium formation and initial penetration.

\section{DISCUSSION}

Fungal pathogens appear to favor one mode of high-affinity iron acquisition over the other. The mammalian pathogen $A$. fumigatus requires siderophores but not RIA for full virulence (Schrettl et al. 2004, 2007). The same has been demonstrated for the necrotroph $F$. graminearum in its interaction with maize (Greenshields et al. 2007) and implied for Epichlö̈ festucae in its mutualistic interaction with ryegrass (Johnson et al. 2013). The biotroph U. maydis, on the other hand, requires RIA for virulence, whereas siderophores are dispensable (Eichhorn et al. 2006; Mei et al. 1993). The necrotroph C. heterostrophus was previously shown to require extracellular siderophores for full virulence to maize (Oide et al. 2006). Because RIA ftrl, fet 3 , and fet3ftrl mutants have no abnormal

Table 3. Quantitative analysis of Cochliobolus heterostrophus intra- and extracellular siderophores

\begin{tabular}{lrrll}
\hline Siderophore & Wild type $^{\mathbf{a}}$ & \multicolumn{1}{c}{ nps $^{\mathbf{a}, \mathbf{b}}$} & nps $^{\mathbf{a}, \mathbf{b}}$ & nps2nps $^{\mathbf{b}}$ \\
\hline Broth (mg/liter) & & & & \\
Neocoprogen II & $2.76(0.8)$ & $5.50(0.85)$ & nd & nd \\
Neocoprogen I & $11.91(6.21)$ & $32.86(8.05)$ & nd & nd \\
Coprogen & $4.14(2.87)$ & $27.07(8.04)$ & nd & nd \\
Mycelium (mg/g of dry weight) & & & \\
Ferricrocin & $0.82(0.12)$ & nd & $1.60(0.39)$ & nd \\
Neocoprogen II & $0.98(0.42)$ & $1.10(0.29)$ & nd & nd \\
Neocoprogen I & $5.45(1.78)$ & $7.71(2.02)$ & nd & nd \\
Coprogen & $2.06(0.62)$ & $6.18(1.68)$ & nd & nd \\
\hline
\end{tabular}

${ }^{\mathrm{a}}$ Value in parentheses $=$ standard error.

${ }^{\mathrm{b}}$ nd $=$ none detected. phenotypes, we can conclude that $C$. heterostrophus prefers high affinity siderophore iron acquisition over RIA.

In contrast to RIA single mutants, however, the combined RIA and siderophore mutants nps6ftrl, nps6fet3, and nps6fet3ftrl are all impaired in growth without supplemental iron, indicating that the RIA mechanism is an important irongathering backup system when extracellular siderophores are absent. Note that deletion of FTR1, FET3, or FTR1 and FET3 was sufficient to disrupt RIA. Growth was further disrupted in nps2nps6ftr1, suggesting that iron acquisition and storage both contribute to proper iron homeostasis.

Removal of both RIA and extracellular siderophores impairs growth and asexual development and only by supplying high quantities of iron (much higher than would be found naturally) can growth be restored. Neither nps 6 nor ftrl mutants have this phenotype, suggesting that one iron acquisition system can substitute for the other in vitro (but not in planta). Unlike NPS6, FTR1 expression was not repressed by the iron-responsive transcription factor Sre1 (Zhang et al. 2013). This, coupled with our finding that ftrl phenotypes occur only in the absence of NPS6, supports a model wherein the extracellular siderophore coprogen is favored for high-affinity iron acquisition. The RIA system functions well enough to allow growth of nps 6 mutants in standard culture but not in the presence of iron or oxidative stress or in planta. Further removal of intracellular siderophores (ferricrocin) results in extremely limited growth without supplementation, likely due to an already ironlimited fungus being extra-sensitive to disruption of iron and oxidative stress homeostasis.

There is only one other study thus far that we are aware of in which RIA/siderophore double mutants were generated (Schrettl et al. 2004). A. fumigatus sidA/ftrA double mutants lacked RIA plus extracellular and intracellular siderophores because SidA is required for biosynthesis of both types of siderophore. These mutants were highly defective in growth, as we have demonstrated here for C. heterostrophus nps 2 nps6ftrl. Our study is unique, however, in distinguishing between extracellular and intracellular siderophore mutations, in combination with RIA mutations (i.e., we generated nps 2 ftrl, nps6ftrl, and nps2nps6 ftrl mutants). Interestingly, loss of intracellular siderophores and RIA (nps 2 ftrl) does not affect stress sensitiv-

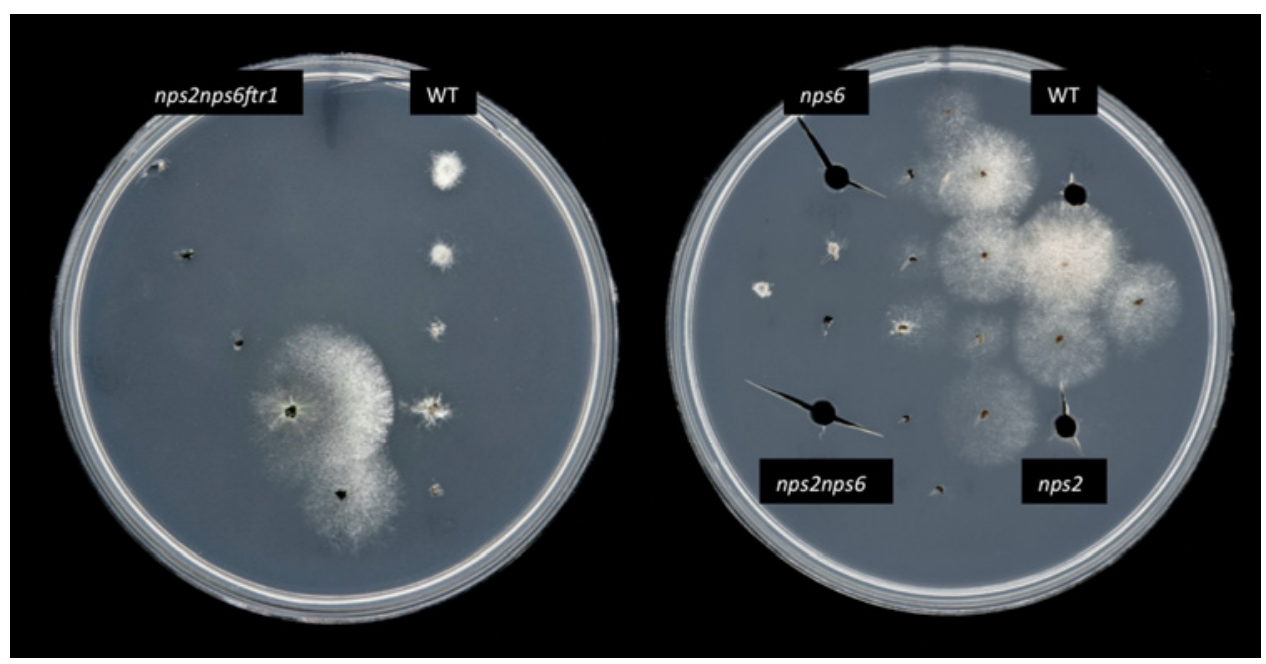

Fig. 6. Growth of nps2nps6ftr1 mutants is restored by proximity to WT and $n p s 2$ strains or filtrates but not by nps6 or nps2nps6 culture filtrates. Left: Small amounts of wild-type (WT) (C4) mycelia with conidia (without agar medium) were placed in a vertical line on the right side of a plate of complete medium without salt solution (CMNS) + hygromycin B. WT is unable to grow because it is sensitive to hygromycin B. Similar amounts of hygromycin B-resistant triple mutant nps2nps6ftrl (strain Ch1731-T6-3) were placed on the diagonal with patches progressively closer to the WT strain. The nps2nps6ftrl mutant is unable to grow unless it is in close proximity to WT, in which case it grows towards the WT culture on the plate. Right: When $n p s 2 n p 6 f t r 1$ is grown adjacent to filtrate from WT, nps6 (strain Chnps6-1), nps2 (strain Chnps2-1), and nps2nps6 (strain Ch1495-T1-1) liquid cultures, only WT and nps2 filtrate can restore growth. 
ity, growth, or virulence. In the presence of a functional NPS6 gene, nps2, ftrl, and nps2ftrl mutants are all WT for these phenotypes.

The oxidative and iron stress findings in this study demonstrate that, although deletion of NPS2 alone has no effect on sensitivity to ROS or to low iron (Oide et al. 2007), deletion of NPS2 has a clear effect when combined with nps6. Growth of nps6ftrl mutants, on the other hand, was not reduced relative to nps6 on media containing oxidative stressors. This confirms that coprogen is sufficient for dealing with both oxidative and iron stress, while ferricrocin but not RIA plays a secondary role.

The nps 2 ftrl mutants were like WT in terms of virulence on maize, demonstrating that $C$. heterostrophus relies first and foremost on extracellular siderophore-mediated iron acquisition for virulence. Both nps6ftrl and nps2nps6 mutants were further reduced in virulence compared with nps6, with nps $2 n p s 6 f t r 1$ mutants almost completely unable to proceed past appressorium formation. The reduction in disease seen for nps6ftrl could be due to its reduced growth without excess iron but is not a product of increased sensitivity to oxidative or iron stress, given its growth on media containing iron or oxidative stressors. Conversely, nps 2 nps 6 mutants are not impaired in in vitro growth but are more sensitive to oxidative stress than $n p s 6$ mutants. This suggests that both iron limitation and oxidative stress may be the cause of the reduced virulence of nps6 strains.

\section{MATERIALS AND METHODS}

Fungal strains and culture conditions.

C. heterostrophus WT race $\mathrm{T}$ strain $\mathrm{C} 4$ (Tox1+; MAT1-2, American Type Culture Collection [ATCC] number 48331) was used for all transformations and strain C2 (Toxl+; MAT1-1; alb1, ATCC 48329) was used as an albino tester for initial crosses. All strains used in this study are listed in Table 1. Strains were stored at $-80^{\circ} \mathrm{C}$ in liquid CM (Leach et al. 1982) containing $25 \%$ glycerol and were plated onto CMX (Tzeng et al. 1992) for growth and optimal conidiation. Fungi were grown at $24^{\circ} \mathrm{C}$ in an alternating 16 -h-light and 8-h-dark cycle
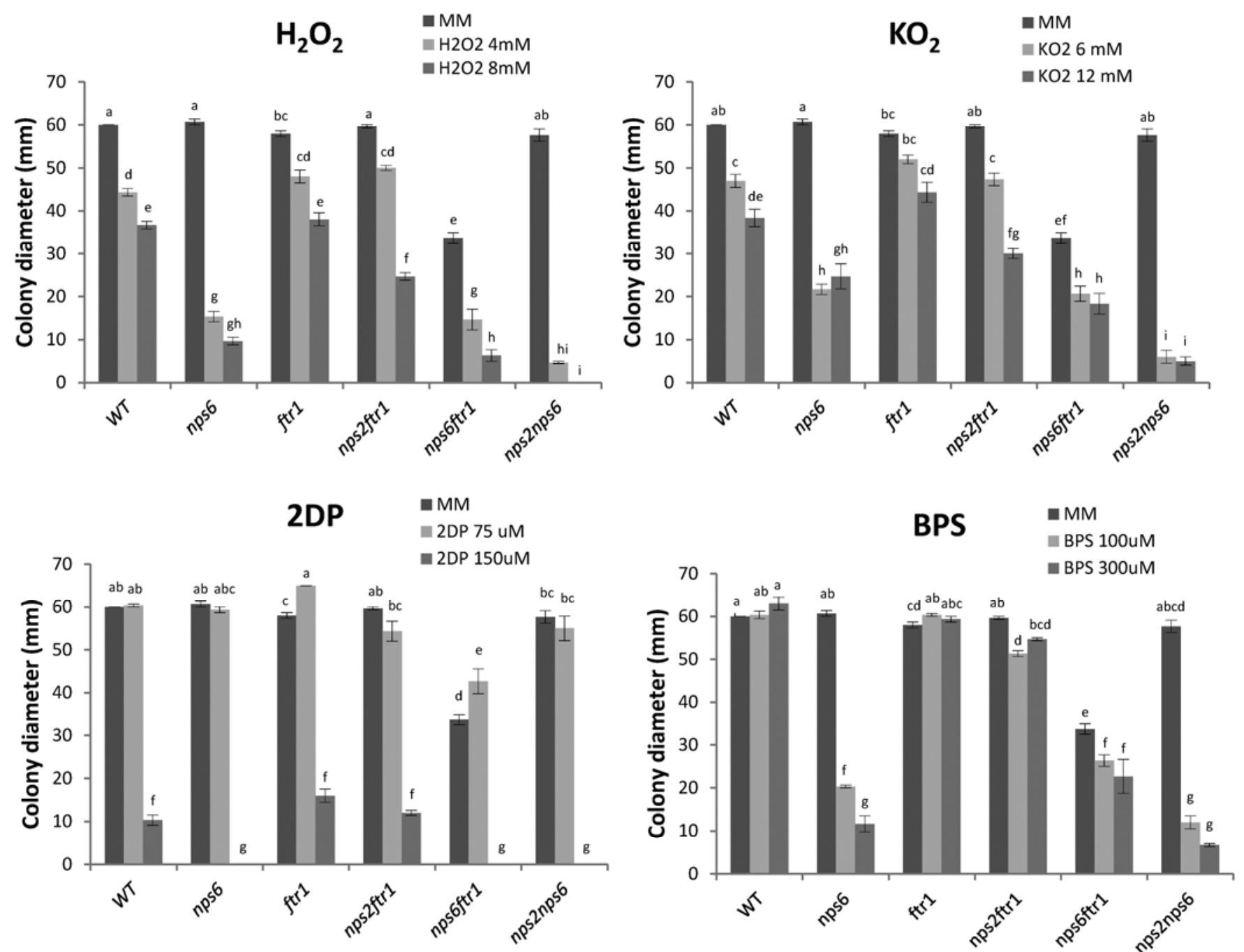

Fig. 7. Iron acquisition and storage mutants are sensitive to oxidative and iron stress. Wild type (WT) (strain C4), nps6 (strain Chnps6-1), ftr1 (strain Chftr11), nps2ftr1 (strain Ch1723-T2-5), nps6ftr1 (Ch1722-R1), and nps2nps6 (strain Ch1495-T1-1) were plated on minimal medium (MM) containing increasing concentrations of the oxidative stressors $\mathrm{H}_{2} \mathrm{O}_{2}$ and $\mathrm{KO}_{2}$, the membrane permeable iron chelator 2,2-dipyridyl (2DP), and membrane impermeable iron chelator bathophenanthroline disulfonate (BPS). Average colony diameter was measured from leading edge to leading edge of hyphal growth. The ftrl and nps 2 ftr1 mutants are similar to WT in response to all stressors, suggesting lack of intracellular siderophores and the reductive iron assimilation mechanism does not impact resistance to these stresses. As reported previously (Oide 2007), nps6 is more sensitive than WT and nps2nps6 is more sensitive than nps6 on $\mathrm{H}_{2} \mathrm{O}_{2}$ and $\mathrm{KO}_{2}$. In these experiments, nps6 and nps2nps6 responded similarly to iron stress, however stress conditions chosen were not optimal for a distinction to be drawn. The growth of nps6ftr1 mutants is comparable to that of nps6 on both types of oxidative stress and on BPS, although its reduced growth on MM is a complicating factor. Within a graph, different letters above bars indicate values that are statistically significantly different as determined by one-way analysis of variance and Tukey's range test. 
under fluorescent light (Watt-Miser F34 WW/RS/WM, Warm White; General Electric, Home Depot, Ithaca, NY, U.S.A.).

MM (Leach et al. 1982) was used for iron and oxidative stress assays. CMNS was used for selection for resistance to hygromycin $\mathrm{B}$. $\mathrm{CMX}+\mathrm{Fe}$ and $\mathrm{MM}+\mathrm{Fe}$ were $\mathrm{CMX}$ or $\mathrm{MM}$ with $100 \mu \mathrm{M}$ ferric citrate (Sigma, St. Louis) added prior to autoclaving, unless stated otherwise. Sach's (Luttrell 1958) medium or MM (Oide et al. 2007) was used for crosses.

\section{Iron and fungal growth.}

Iron source supplementation was tested on solid CMX with ferric citrate, ferrous sulphate $7 \cdot \mathrm{H}_{2} \mathrm{O}$ (Mallinckrodt), and ferric chloride $6 \cdot \mathrm{H}_{2} \mathrm{O}$ (Fluka). Iron, from aliquots of $1 \mathrm{mM}$ sterilized stock solutions, was added to CMX after autoclaving to final concentrations of $0.25,2.5$, and $25 \mu \mathrm{M}$. Cultures were grown for 6 days in the dark and were then photographed.
For liquid cultures, spore suspensions were prepared by applying 2 to $3 \mathrm{ml}$ of $\mathrm{CM}$ to culture plates, rubbing the surface to dislodge conidia, and filtering the slurry through sterile cheesecloth. Conidia $\left(10^{5}\right)$ were added to $10 \mathrm{ml}$ of liquid CM, with or without $100 \mu \mathrm{M}$ ferric citrate. Cultures were grown for $18 \mathrm{~h}$ at room temperature at $150 \mathrm{rpm}$, and aliquots were observed under a Leica DM5500 Epifluorescence microscope using differential interference contrast at the Boyce Thompson Institute Plant Cell Imaging Center (Ithaca, NY, U.S.A.). Images were acquired using Qcapture Pro 6.0 (Qimaging, Surrey, Canada).

\section{Identification of RIA associated genes.}

Orthologs of the S. cerevisiae iron permease FTR1 and multicopper oxidase FET3 in the RIA pathway (Table 1) were identified initially by BLAST search (Altschul et al. 1990), using the $S$. cerevisiae proteins as a query against the $C$. heter-

A
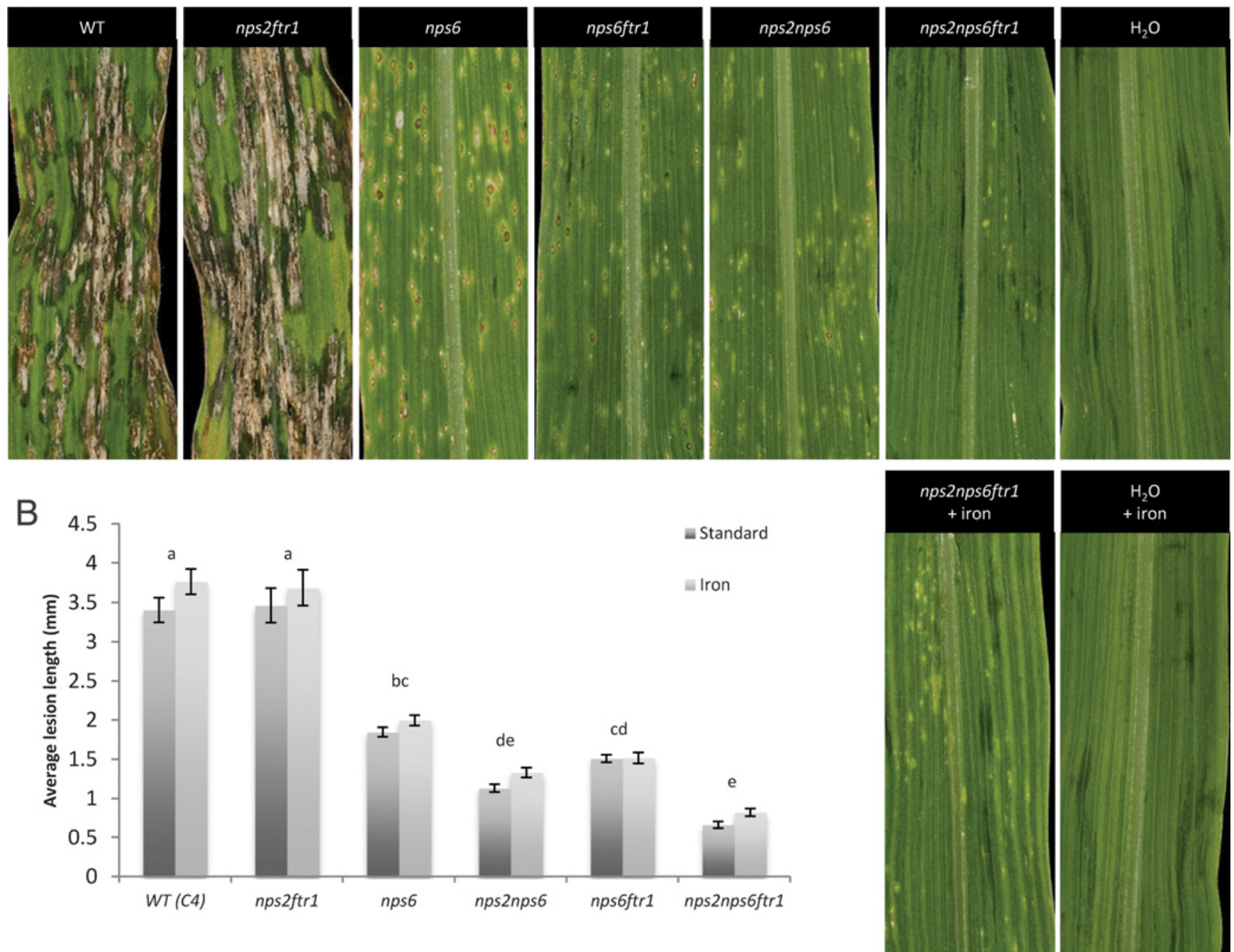

Fig. 8. FTR1 and NPS2 affect virulence in a manner secondary to NPS6. A, Representative fourth true leaves from N-cytoplasm W64-A corn 5 days after inoculation with wild type (WT) and iron mutants are shown. On leaves inoculated with WT (strain C4) and nps2ftrl (strain Ch1723-T2-5), lesions are dark, long, and necrotic. The nps6 (strain Chnps6-1) mutants cause smaller brown lesions, while nps6ftrl (strain Ch1722-R1) and nps2nps6 (strain Ch1495-T1-1) cause much smaller, lighter lesions with small dark centers. The nps2nps6ftrl (strain Ch1731-T6-3) mutants cause the smallest lesions, observable as white flecks. All leaf images are applications without supplemental iron, with the exception of the control water-inoculated leaves and the triple mutant-inoculated leaves, which are shown without and with supplemental iron. B, Average lesion size of fourth true leaves from N-cytoplasm W64-A maize inoculated with WT and iron mutants. The nps2ftrl (and ftrl, not shown) mutant lesion sizes are like WT. As shown previously, nps6 mutants are reduced in virulence and nps2nps 6 mutants are further reduced. The nps6ftrl mutant is comparable to nps2nps6, while nps2nps6ftrl mutants are extremely reduced in virulence compared with WT. Iron supplementation slightly increased lesion size for all inoculated plants. This difference was not significantly different as determined by one-way analysis of variance (ANOVA) and Tukey's range test for any mutant or WT. Letters above bars indicate strains that were statistically significantly different for standard (lacking supplemental iron) treatments as determined by one-way ANOVA and Tukey's range test. Means not connected by the same letter are significantly different. 
ostrophus C5 gene catalog (Condon et al. 2013). FTR1 (JGI identification number 104817, 1,778 bp) and FET3 (JGI identification number $104814,2,030 \mathrm{bp}$ ) were both located on scaffold 3 , separated by $1,778 \mathrm{bp}$. Deletions were made in the sister inbred strain $\mathrm{C} 4$.

DNA manipulations and fungal transformations.

Fungal genomic DNA was prepared using the Ultraclean Microbial DNA isolation kit (MO BIO, Carlsbad, CA, U.S.A.). PCR reactions were carried out with Phusion (New England Biolabs, Ipswich, MA, U.S.A.) DNA polymerase and mix for generating transformation constructs or GoTaq (Promega, Madison, WI, U.S.A.) for screening mutants, following the manufacturer's recommendations.

Transformations were performed using split marker-based homologous integration (Catlett et al. 2003; Turgeon et al. 2010). The 700- to 1,000-bp DNA flanking ( $5^{\prime}$ and $3^{\prime}$ flanks) coding sequences were amplified, using primer pairs with extensions complementary to M13Rhyg for the reverse primer of one flanking region and M13Fhyg for the forward primer of the other flanking region. FTR 1 was deleted using the upstream primer pair ftrupf/ftrupr and the downstream pair ftrdnf/ftrdnr to amplify the $5^{\prime}$ and $3^{\prime}$ flanks, respectively. FET3 was deleted using the upstream pair fet3upf/fet3upr and the downstream pair fet3dnf/fet3dnr, and FTR1 and FET3 were deleted together using the upstream (relative to FET3) pair fet3upf2/fet3upr2 and the downstream pair fet $3 \mathrm{dnf} / \mathrm{fet} 3 \mathrm{dnr}$. NPS 2 and NPS6 gene deletions were described previously (Oide et al. 2006, 2007).
Protoplasting and transformation of strain $\mathrm{C} 4$ was performed as described by Turgeon and associates (2010). Candidate transformants recovered from the hygromycin B overlay were transferred approximately 1 week after plating to fresh selective medium (CMNS containing $50 \mu \mathrm{g}$ hygromycin B per milliliter) to confirm resistance. Conidia were streaked on $5 \%$ water agar to separate individual conidia and eliminate heterokaryons, and individual germinating conidia were transferred to CMX. Purified candidates were then patched onto CMNS hygromycin $\mathrm{B}$ (50 $\mu \mathrm{g}$ hygromycin B per milliliter) and CMX to confirm antibiotic resistance and for storage in glycerol, respectively.

DNA was prepared from candidate transformants and was subjected to diagnostic PCR to verify gene deletion. A set of primers internal to the deleted gene and two sets of primer pairs, in which one primer was internal to the introduced selectable marker and the other was external to either the $5^{\prime}$ or the $3^{\prime}$ flanking region used to delete the gene, were used. DNA from candidates with a gene of interest deleted and replaced with the selectable marker showed a band of predictable size when a primer external to the $5^{\prime}$ or the $3^{\prime}$ flanking region was combined with either NLC37 or NLC38 (internal to $\mathrm{HygB}$ ), while WT DNA did not. Additionally, a band was amplified from WT DNA when a primer pair internal to the gene targeted for knockout was used, whereas no band was amplified from candidate deletion mutant DNA.

The ftrl candidates were confirmed using internal primer pair ftrinf/ftrinr to verify gene deletion and external primer pairs fet3upr/NLC37 and fet3extfarupf/NLC38 to verify cor-
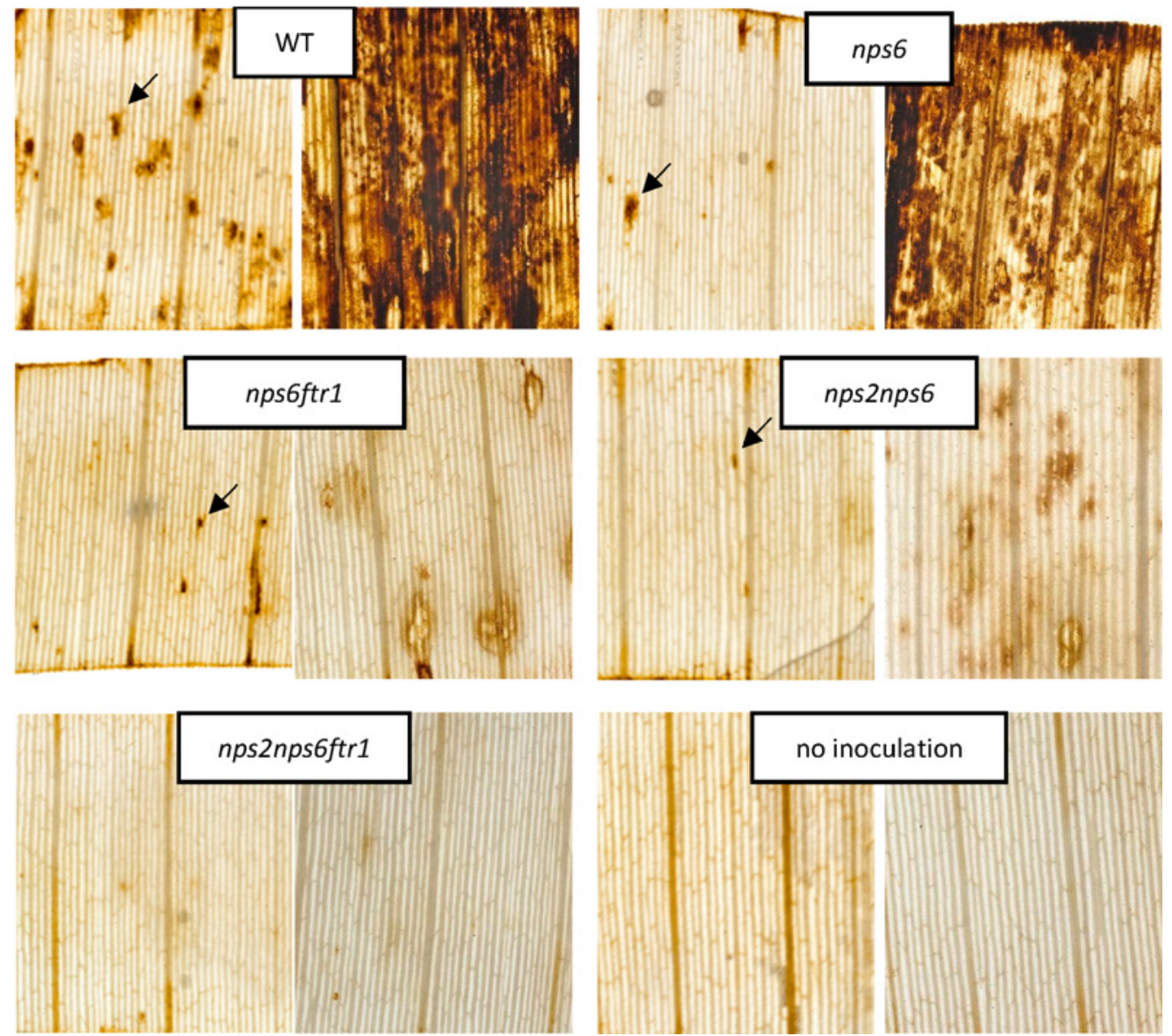

Fig. 9. Reactive oxygen species production as indicated by 3,3'-diaminobenzidine (DAB) staining is reduced in maize leaves inoculated with iron mutants. Inoculated and DAB-stained leaves at 1 (left in each pair) and 5 (right in each pair) days after inoculation. In wild type (strain C4) and $n p s 6$-challenged (strain ChChnps6-1) leaves, DAB precipitates form at discrete locations after $24 \mathrm{~h}$ (arrow) and ubiquitously throughout the leaf by 5 days. The $n p s 6 f t r 1$ (strain Ch1722-R1) and nps2nps6 (strain Ch1495-T1-1) mutants form fewer localized DAB precipitates at 24 h and much smaller, contained DAB-stained areas at 5 days. The nps2nps6ftrl (strain Ch1731-T6-3) mutants do not form visible DAB precipitate at 1 or 5 days. 

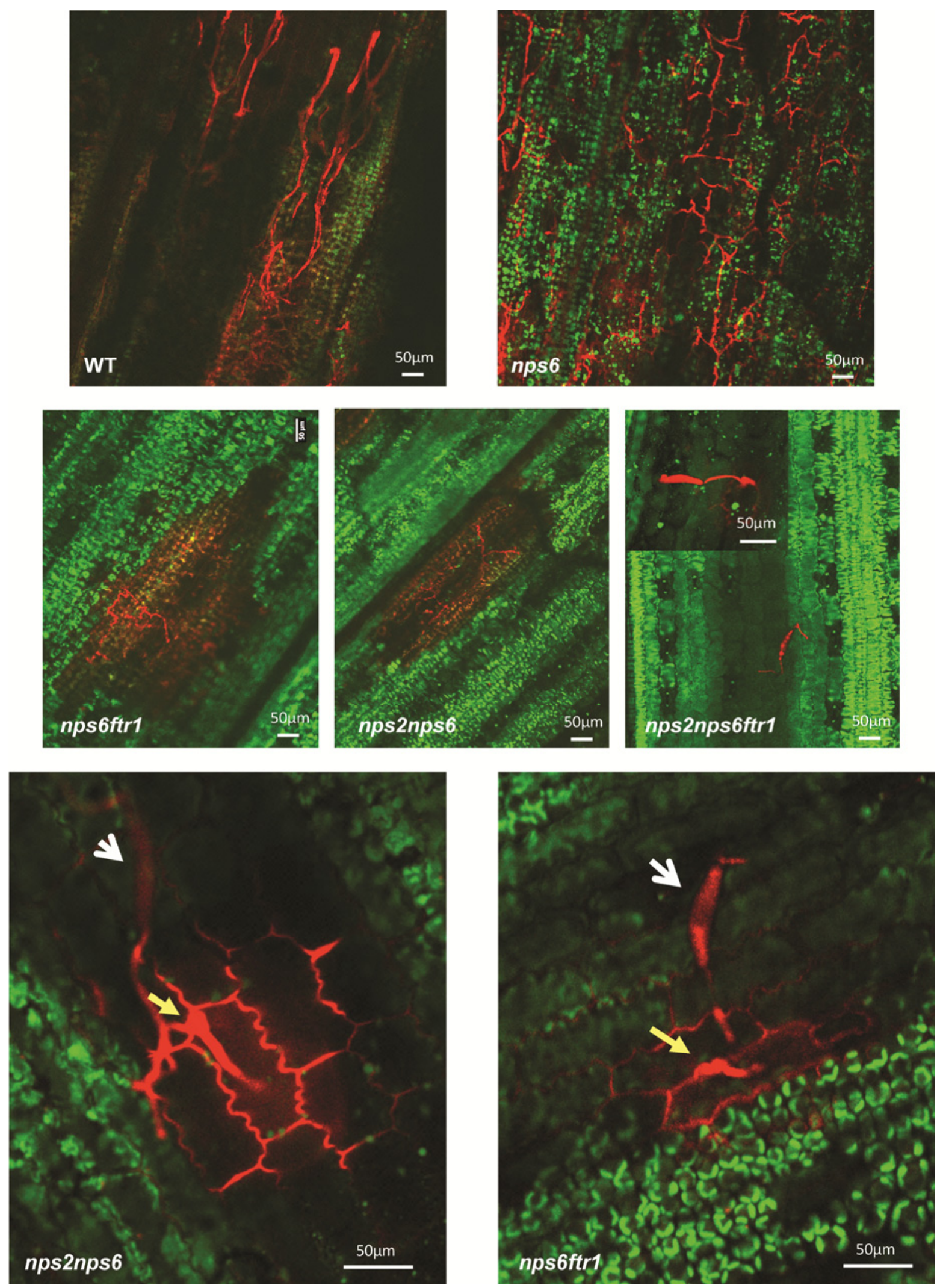

Fig. 10. Iron mutants are progressively impaired in colonizing maize leaves. Top and middle rows: Dsred-expressing strains of wild type (WT) (strain Ch1761-R7), nps6 (strain Ch1762-T1-1), nps6ftr1 (strain Ch1764-T1-1), nps2nps6 (strain Ch1765-T2-3), and nps2nps6ftr1 (strain Ch1766-R2) mutants were inoculated onto maize and leaves were visualized by confocal microscopy at 5 days. The scale bar is set to $50 \mu \mathrm{m}$ and is equivalent for each image. WT and nps6 mutants extensively colonized the leaf; nps6ftrl and nps2nps6 growth was less prolific and caused smaller autofluorescent patches of dead plant tissue; nps2nps6ftrl was able to germinate and form appressoria (insert image taken at $24 \mathrm{~h}$ ) but did not typically penetrate or grow inside the leaf. When it did, it did not progress beyond the initial cell colonized. Bottom row: At $24 \mathrm{~h}$, all strains had attached and formed appressoria (only nps2nps6 and nps6ftrl shown). Conidia are still attached (open white arrows) and the precise site of penetration can be identified (closed arrows). Autofluorescence at the perimeter of penetrated epidermal and surrounding cells occurs in an emission spectrum indistinguishable from that of Dsred. 
rect integration into the $5^{\prime}$ and the $3^{\prime}$ target flanks, respectively. The fet 3 candidates were confirmed using the internal primer pair fet3inf/fet3inr and the external primer pairs fet3extupf/ NLC38 and fet3extdnr/NLC37. The fet 3 and ftrl double deletion candidates were confirmed using the internal primer pair fet3inf/fet3inr and the external primer pairs fet3extdnr/NLC37 and fet3extfarupf/NLC38.

\section{Construction of combinatorial mutants.}

Transformants of strain C4 (MAT1-2) were initially crossed to strain $\mathrm{C} 2$ (MAT1-1) to generate albino and pigmented strains of both mating types. All WT genes were replaced with the hygB gene, conferring resistance to hygromycin B. Tracking hygromycin $\mathrm{B}$ resistance, therefore, also tracked gene deletion (assuming hyg $B$ has correctly replaced the targeted gene, as confirmed by diagnostic PCR of candidate transformants). Single and polymutants were recovered from crosses by collecting random or tetrad progeny. For tetrads, the full set of progeny from a given ascus was collected, in isolation from other asci to ensure each ascospore was from the same ascus. In crosses between two hygR parents, if hygromycin B-sensitive progeny are observed in a tetrad, the tetrad must contain WT progeny and combinatorial mutants containing each mutation carried by both parents. Diagnostic PCR reactions with the same primer pairs used to confirm successful gene deletions were used to verify the genotype of mutants with multiple gene deletions collected from both random and tetrad isolated ascospores.

nps2nps6 strains were tetrad progeny of Chnps2-1 $\times$ Chnps61R2 crosses and nps 2 ftrl strains were tetrad progeny of Chftr1-1 × 1449-T1-5 crosses. Additional nps2ftrl strains were generated by crossing the nps $2 f t r 1$ strain Ch1723-T2-5 to WT (CB11) and collecting whole tetrads. The nps6fet 3 strains were random progeny from Chnps6-1-R $2 \times$ Chfet3-1 crosses; nps6fet3ftr1 strains were random progeny of Chnps6-1R2 $\times$ Chfet3ftr1-1 crosses; nps6ftr1 strains were random progeny of Chnps6-1R2 $\times$ Chftr1-1; nps2nps6ftrl were tetrad progeny of nps6ftrl strain $1722-\mathrm{R} 1 \times$ Chnps2-1 and ftrl strain $1721-\mathrm{R} 3 \times$ nps2nps6 strain 1495-T1-6 (Table 2).

\section{Evaluation of fertility.}

Crosses (Table 2) were set up using autoclaved senescent maize leaves placed on MM as described previously (Oide et al. 2007). Crosses between WT strains C4 (MAT1-2) and either C2 (for $n p s 2, n p s 6, n p s 2 n p s 6$ ) or C9 (for ftr1, nps2ftr1, nps6ftr1) were set up as a control for all experiments. Fertility was evaluated based on the average number of pseudothecia per square centimeter of senescent corn leaf and the average number of asci per pseudothecium.

\section{Asexual development.}

Asexual sporulation of WT (C4) and nps6 (Chnps6-1), ftr1 (Chftr1-1), nps6ftrl (Ch1722-R1), nps2ftrl (Ch1723-T2-5), nps2nps6 (Ch1495-T1-6), and nps2nps6ftrl (Ch1731-T6-3) mutant strains was quantified. Strains were grown on CMX with and without exogenous application of iron $(100 \mu \mathrm{M}$ ferric citrate) for 7 days at $24^{\circ} \mathrm{C}$ under $16 \mathrm{~h}$ of light and $8 \mathrm{~h}$ of dark fluorescent light and were photographed. The entire area of each plate was scraped with $0.2 \%$ Tween 20 in $\mathrm{H}_{2} \mathrm{O}$ to collect conidia and the slurry was filtered through sterile cheesecloth and was suspended in $10 \mathrm{ml}$ of $0.2 \%$ Tween 20 . The concentration was determined by counting spores under a stereomicroscope with a haemocytometer and the number per plate was estimated for each plate.

\section{Feeding plates.}

Triple high-affinity iron mutant nps2nps6ftrl (Ch1731-T6-3 or Ch1743-T6-1) was grown for 5 days on $\mathrm{CMX}+100 \mu \mathrm{M}$ ferric citrate. Small scrapings of the culture (approximately $1 \times$ $1 \mathrm{~mm}$ ) were transferred to CMNS with hygromycin B. Care was taken to collect mycelium and conidia only, without any of the iron-rich growth medium. For experiments with the WT hygromycin B-sensitive culture, scrapings were set up in a vertical row on one side of the plate and the nps 2 nps $6 f t r 1$ triple mutant scrapings were placed on a diagonal line opposite, with each scraping progressively closer to the WT row.

When supplementing with hygromycin B-resistant strains $n p s 2, n p s 6$, or nps2nps6, culture filtrate was used instead of mycelial scrapings, to prevent the supplementing culture from overgrowing the nps2nps6ftrl mutant. For this, flasks containing $50 \mathrm{ml}$ of $\mathrm{CM}$ were inoculated with a $5 \times 5 \mathrm{~mm}$ aliquot of scraped conidia and were shaken overnight. Cultures were centrifuged for $5 \mathrm{~min}$ at 10,000 RPM at room temperature in a tabletop centrifuge to pellet mycelium. A $20-\mu 1$ supernatant was transferred to wells in the CMNS with hygromycin B plates carved into the agar with a cork borer. Plates were grown for 2 days at $24^{\circ} \mathrm{C}$ under a 16-h-light and 8-h-dark alternating fluorescent light cycle and were photographed.

\section{Isolation, identification, and quantitative analysis of siderophores.}

Fungal cultures for HPLC analyses were grown and prepared as described previously (Oide et al. 2006, 2007). Quantitative analysis of siderophore production was performed as described previously (Oide et al. 2006). Coprogen and ferricrocin, used as reference standards, were purified as described (Oide et al. 2006, 2007).

\section{Stress sensitivity assays.}

Sensitivity to $\mathrm{H}_{2} \mathrm{O}_{2}$, the superoxide-generator $\mathrm{KO}_{2}$, the membrane-permeable iron chelator 2DP, and the membrane-impermeable iron chelator BPS was assessed. A fresh stock solution of each stress agent was prepared for each experiment (1 M $\mathrm{KO}_{2}, 10 \mathrm{mM}$ 2DP, and $100 \mathrm{mM}$ BPS solutions in Millipore water), and the stress agents were added to MM after autoclaving and cooling to approximately $48^{\circ} \mathrm{C}$. Fresh MM plates with the stress agents were prepared for each experiment. All experiments were carried out in the dark at room temperature and were allowed to grow for 6 days before being photographed. Colony diameters were measured from leading tip to leading tip of hyphal growth; the thickness of growth within that diameter was not considered. Each strain was plated on each condition in triplicate, and the entire experiment was repeated three times.

\section{Plant cultivars and growth conditions.}

Corn cultivar W64A with $\mathrm{N}$ cytoplasm was used in this study. Corn was grown in a growth chamber under a 16-h-light and 8 -h-dark cycle at $24^{\circ} \mathrm{C}$. Seed was planted three plants per \#6 standard pot, in Cornell Mix soil $(3 \times 3.8$ cubic feet compressed bales of peat moss, $2 \times 6$ cubic feet bags of vermiculite, 20 pounds of dolomitic limestone, 6 pounds 11-5-11 fertilizer; Cornell University New York State Agricultural Experiment Station website).

\section{Virulence.}

Virulence assays of $C$. heterostrophus on maize were carried out after emergence of the fourth true leaf (approximately three weeks after planting), as described previously (Oide et al. 2006). At least three replicates (i.e., inoculation to three independent plants) were set up for each strain and experiments were repeated at least three times in all cases.

All fungal strains were recovered from $-80^{\circ} \mathrm{C}$ glycerol stocks on CMX with $100 \mu \mathrm{M}$ supplemental ferric citrate (if experiment included nps6ftrl or nps2nps6ftrl; otherwise, plain CMX was used) for inoculation. After strains were grown for 
10 to 14 days, conidia were harvested by scraping plates in $0.02 \%$ Tween 20 and filtering through cheesecloth to remove hyphal debris. Conidia were counted with a haemocytometer and were adjusted with $0.02 \%$ Tween 20 to $2 \times 10^{4}$ conidia per milliliter. Conidia were spray-inoculated onto maize plants using Preval sprayers, at the rate of $2 \mathrm{ml}$ per plant. Plants were placed overnight in a mist chamber and were then moved to the growth chamber. Third or fourth true leaves were collected after 5 days and were photographed. At least 50 independent lesions were measured for each strain for each experiment. Statistical analyses were performed using one-way analysis of variance with a Tukey's range test using JMP software (SAS Institute, Cary, NC, U.S.A.).

Viability of nps2nps6ftrl conidia was confirmed by harvesting conidia as above and placing 1-ml aliquots of spore suspension on glass microscope slides. Conidia were incubated in a moist chamber for $90 \mathrm{~min}$, and germination rates were counted for 50 conidia, each, on three glass slides. The entire experiment was carried out twice. WT conidia were used as a positive control.

For iron supplementation, maize leaves were rubbed with 1 $\mathrm{mM}$ ferric citrate solution containing $1 \mathrm{~g}$ of glucopon $215 \mathrm{CS}$ UP (Fluka, Milwaukee, WI, U.S.A.) per liter, $1 \mathrm{~h}$ prior to inoculation. The iron-treated plants were incubated in a growth chamber under continuous light until inoculation. Fungal inoculation was carried out in the same way as the standard inoculation assay, except that spore suspensions with or without 0.5 $\mathrm{mM}$ ferric citrate were prepared. Control plants inoculated with $0.5 \mathrm{mM}$ ferric citrate without fungal spores were set up as a control. A fresh stock solution of ferric citrate (10 $\mathrm{mM}$ in water) was prepared for each experiment.

\section{DAB staining and confocal microscopy.}

Inoculation and growth conditions for confocal microscopy and DAB staining were the same as above, except fungal spores were as concentrated as possible. For this, conidia from an entire $100 \times 15$ petri dish were scraped into $50 \mathrm{ml}$ of $0.02 \%$ Tween 20 in $\mathrm{H}_{2} \mathrm{O}$, and all $50 \mathrm{ml}$ was used per inoculation. For DAB staining, leaf squares were submerged in DAB solution $(1 \mathrm{mg} / \mathrm{ml}$, pH 3.7), were shaken for $4 \mathrm{~h}$ at $50 \mathrm{rpm}$, were then transferred to $3: 1$ ethanol/acetic acid, and were shaken overnight at $50 \mathrm{rpm}$ to clear leaves.

Prior to confocal microscopy, fungal mutants were crossed to a red fluorescent Dsred (Matz et al. 1999) strain previously transformed using the vector pIGREDPAPA (a modification of pIGPAPA) (Horwitz et al. 1999), with the open reading frame of Dsred replacing green fluorescent protein. Tetrads were collected from nps6, nps $2 n p s 6$, and nps6ftr $1 \times$ Dsred crosses; random progeny were collected for WT $\times$ Dsred and nps $2 n p s 6 f t r 1 \times$ Dsred crosses. Progeny were assayed for fluorescence under a Leica DM5500 Epifluorescence microscope, and were screened for hygromycin B resistance on CMNS with hygromycin B (50 $\mu \mathrm{g} / \mathrm{ml}$ ) plates. Complete tetrads that contained hygromycin Bsensitive, nonfluorescent progeny (and, therefore, the intended fluorescent, hygB-resistant mutant in the same ascus) were screened by PCR to verify genotype. Fluorescent, $h y g B^{R}$ nps2nps6ftr1 progeny were collected by phenotype (inability to grow on MM and CMX without supplemental iron) and fluorescence.

Inoculation was carried out as above for DAB staining. Fourth leaves were collected 1 day $(24 \mathrm{~h})$ and 5 days after inoculation and were trimmed with a scalpel to approximately $1 \times$ $1 \mathrm{~mm}$ squares along either side of the major vein. For microscopy, leaves were examined in a Leica TCS SP5 laser scanning confocal microscope at the Boyce Thompson Institute Plant Cell Imaging Center. Images were collected by exciting samples at $488 \mathrm{~nm}$ with an argon laser and collecting emissions at 566 $\mathrm{nm}$ (Dsred) and $675 \mathrm{~nm}$ (chloroplasts). Images were processed in ImageJ (Schneider et al. 2012).

\section{ACKNOWLEDGMENTS}

The support of the United States Department of Agriculture National Institute of Food and Agriculture grant number 2010-65108-20500 to B. G. Turgeon and Binational Agricultural Research and Development Fund (BARD) grant IS 4167-08C to B. A. Horwitz and B. G. Turgeon is gratefully acknowledged.

\section{LITERATURE CITED}

Aguirre, J., Ríos-Momberg, M., Hewitt, D., and Hansberg, W. 2005. Reactive oxygen species and development in microbial eukaryotes. Trends Microbiol. 13:111-118

Altschul, S. F., Gish, W., Miller, W., Myers, E. W., and Lipman, D. J. 1990. Basic local alignment search tool. J. Mol. Biol. 215:403-410.

Birch, L. E., and Ruddat, M. 2005. Siderophore accumulation and phytopathogenicity in Microbotryum violaceum. Fungal Genet. Biol. 42:579589.

Bronson, C. R. 1988. Ascospore abortion in crosses of Cochliobolus heterostrophus heterozygous for the virulence locus Tox1. Genome 30:1218.

Catlett, N. L., Lee, B. N., Yoder, O. C., and Turgeon, B. G. 2003. Splitmarker recombination for efficient targeted deletion of fungal genes. Fungal Genet. Newsl. 50:9-11.

Condon, B. J., Leng, Y., Wu, D., Bushley, K. E., Ohm, R. A., Otillar, R., Martin, J., Schackwitz, W., Grimwood, J., MohdZainudin, N., Xue, C., Wang, R., Manning, V. A., Dhillon, B., Tu, Z. J., Steffenson, B. J., Salamov, A., Sun, H., Lowry, S., LaButti, K., Han, J., Copeland, A. C., Lindquist, E., Barry, K., Schmutz, J., Baker, S. E., Ciuffetti, L. M., Grigoriev, I. V., Zhong, S., and Turgeon, B. G. 2013. Comparative genome structure, secondary metabolite, and effector coding capacity across Cochliobolus pathogens. PLoS Genet 9:e1003233. Published online.

Eichhorn, H., Lessing, F., Winterberg, B., Schirawski, J., Kamper, J., Muller, P., and Kahmann, R. 2006. A ferroxidation/permeation iron uptake system is required for virulence in Ustilago maydis. Plant Cell 18:3332-3345.

Fenton, H. J. H. 1894. Oxidation of tartaric acid in presence of iron. J. Chem. Soc., Trans. 65:899-910.

Finking, R., and Marahiel, M. A. 2004. Biosynthesis of nonribosomal peptides. Annu Rev Microbiol 58:453-488.

Foyer, C. H., and Noctor, G. 2005. Redox homeostasis and antioxidant signaling: A metabolic interface between stress perception and physiological responses. Plant Cell 17:1866-1875.

Greenshields, D. L., Liu, G., Feng, J., Selvaraj, G., and Wei, Y. 2007. The siderophore biosynthetic gene SID1, but not the ferroxidase gene FET3, is required for full Fusarium graminearum virulence. Mol. Plant Pathol. 8:411-421.

Haas, H., Eisendle, M., and Turgeon, B. G. 2008. Siderophores in fungal physiology and virulence. Annu. Rev. Phytopathol. 46:149-187.

Hissen, A. H. T., Wan, A. N. C., Warwas, M. L., Pinto, L. J., and Moore, M. M. 2005. The Aspergillus fumigatus siderophore biosynthetic gene sida, encoding L-ornithine $\mathrm{N}^{5}$-oxygenase, is required for virulence. Infect. Immun. 73:5493-5503.

Horwitz, B. A., Sharon, A., Lu, S.-W., Ritter, V., Sandrock, T. M., Yoder, O. C., and Turgeon, B. G. 1999. A G protein alpha subunit from Cochliobolus heterostrophus involved in mating and appressorium formation. Fungal Genet. Biol. 26:19-32.

Johnson, L. 2008. Iron and siderophores in fungal-host interactions. Mycol. Res. 112:170-183.

Johnson, L. J., Koulman, A., Christensen, M., Lane, G. A., Fraser, K., Forester, N., Johnson, R. D., Bryan, G. T., and Rasmussen, S. 2013. An extracellular siderophore is required to maintain the mutualistic interaction of Epichlö̈ festucae with Lolium perenne. PLoS Pathog 9:e1003332. Published online.

Kaplan, C. D., and Kaplan, J. 2009. Iron acquisition and transcriptional regulation. Chem. Rev. 109:4536-4552.

Kieu, N. P., Aznar, A., Segond, D., Rigault, M., Simond-Cote, E., Kunz, C., Soulie, M.-C., Expert, D, and Dellagi, A. 2012. Iron deficiency affects plant defence responses and confers resistance to Dickeya dadantii and Botrytis cinerea. Mol. Plant Pathol. 13:816-827.

Kosman, D. J. 2003. Molecular mechanisms of iron uptake in fungi. Mol. Microbiol. 47:1185-1197.

Kosman, D. J. 2010. Redox cycling in iron uptake, efflux, and trafficking. J. Biol. Chem. 285:26729-26735.

Kotchoni, S. O., and Gachomo, E. W. 2006. The reactive oxygen species network pathways:an essential prerequisite for perception of pathogen 
attack and the acquired disease resistance in plants. J. Biosci. 31:389-404.

Leach, J., Lang, B. R., and Yoder, O. C. 1982. Methods for selection of mutants and In vitro culture of Cochliobolus heterostrophus. Microbiology 128:1719-1729.

Lee, B.-N., Kroken, S., Chou, D. Y. T., Robbertse, B., Yoder, O. C., and Turgeon, B. G. 2005. Functional analysis of all nonribosomal peptide synthetases in Cochliobolus heterostrophus reveals a factor, NPS6, involved in virulence and resistance to oxidative stress. Eukaryot. Cell 4:545-555.

Lindsay, W. L., and Schwab, A. P. 1982. The chemistry of iron in soils and its availability to plants. J. Plant Nutr. 5:821-840.

Liu, G., Greenshields, D. L., Sammynaiken, R., Hirji, R. N., Selvaraj, G., and Wei, Y. 2007. Targeted alterations in iron homeostasis underlie plant defense responses. J Cell Sci 120:596-605.

Luttrell, E. S. 1958. The perfect stage of Helminthosporium turcicum. Phytopathology 48:281-287.

Matz, M. V., Fradkov, A. F., Labas, Y. A., Savitsky, A. P., Zaraisky, A. G., Markelov, M. L., and Lukyanov, S. A. 1999. Fluorescent proteins from nonbioluminescent Anthozoa species. Nat. Biotechnol. 17:969-973.

Mei, B., Budde, A. D., and Leong, S. A. 1993. sid1, a gene initiating siderophore biosynthesis in Ustilago maydis: Molecular characterization, regulation by iron, and role in phytopathogenicity. Proc. Natl. Acad. Sci. U.S.A. 90:903-907.

Mou, Z., Fan, W., and Dong, X. 2003. Inducers of plant systemic acquired resistance regulate NPR1 function through redox changes. Cell 113:935-944.

Oide, S. 2007. Functional characterization of nonribosomal peptide synthetases in the filamentous ascomycete phytopathogen Cochliobolus heterostrophus. PhD thesis, Cornell University, Ithaca, NY, U.S.A.

Oide, S., Moeder, W., Krasnoff, S., Gibson, D., Haas, H., Yoshioka, K., and Turgeon, B. G. 2006. NPS6, encoding a nonribosomal peptide synthetase involved in siderophore-mediated iron metabolism, is a conserved virulence determinant of plant pathogenic Ascomycetes. Plant Cell 18:2836-2853.

Oide, S., Krasnoff, S. B., Gibson, D. M., and Turgeon, B. G. 2007. Intracellular siderophores are essential for Ascomycete sexual development in heterothallic Cochliobolus heterostrophus and homothallic Gibberella zeae. Eukaryot. Cell 6:1339-1353.

Park, Y.-S., Choi, I.-D., Kang, C.-M., Ham, M.-S., Kim, J.-H., Kim, T.-H., Yun, S.-H., Lee, Y.-W., Chang, H.-I., Sung, H.-C., and Yun, C.-W. 2006. Functional identification of high-affinity iron permeases from Fusarium graminearum. Fungal Genet. Biol. 43:273-282.
Schneider, C. A., Rasband, W. S., and Eliceiri, K. W. 2012. NIH image to ImageJ: 25 years of image analysis. Nat. Methods 9: 671-675.

Schrettl, M., Bignell, E., Kragl, C., Joechl, C., Rogers, T., Arst, H. N., Haynes, K., and Haas, H. 2004. Siderophore biosynthesis but not reductive iron assimilation is essential for Aspergillus fumigatus virulence. J. Exp. Med. 200:1213-1219.

Schrettl, M., Bignell, E., Kragl, C., Sabiha, Y., Loss, O., Eisendle, M., Wallner, A., Arst, H. N., Haynes, K., and Haas, H. 2007. Distinct roles for intra- and extracellular siderophores during Aspergillus fumigatus infection. PLoS Pathog 3:e128-1207. Published online.

Schwarzer, D., Finking, R., and Marahiel, M. A. 2003. Nonribosomal peptides: From genes to products. Nat. Prod. Rep. 20:275-287.

Singh, A., Severance, S., Kaur, N., Wiltsie, W., and Kosman, D. J. 2006. Assembly, activation, and trafficking of the Fet3p-Ftr1p high affinity iron permease complex in Saccharomyces cerevisiae. J. Biol. Chem. 281:13355-13364.

Stearman, R., Yuan, D. S., Yamaguchi-Iwai, Y., Klausner, R. D., and Dancis, A. 1996. A permease-oxidase complex involved in high-affinity iron uptake in yeast. Science 271:1552-1557.

Temme, N., and Tudzynski, P. 2009. Does Botrytis cinerea ignore $\mathrm{H}_{2} \mathrm{O}_{2-}$ induced oxidative stress during infection? Characterization of Botrytis activator protein 1. Mol. Plant-Microbe Interact. 22:987-998.

Turgeon, B. G., Condon, B., Liu, J., and Zhang, N. 2010. Protoplast transformation of filamentous fungi. Methods Mol. Biol. 638:3-19.

Tzeng, T. H., Lyngholm, L. K., Ford, C. F., and Bronson, C. R. 1992. A restriction fragment length polymorphism map and electrophoretic karyotype of the fungal maize pathogen Cochliobolus heterostrophus. Genetics 130:81-96.

Winterbourn, C. C. 1995. Toxicity of iron and hydrogen peroxide: The Fenton reaction. Toxicol. Lett. 82-83:969-974.

Zhang, N., Nurainizzati, M. Z., Scher, K., Condon, B. J., Horwitz, B. A., and Turgeon, B. G. 2013. Iron, oxidative stress, and virulence: Roles of the iron-sensitive transcription factor Sre1 and the redox sensor ChAp1 in the maize pathogen Cochliobolus heterostrophus. Mol. Plant-Microbe Interact. 26:1473-85.

\section{AUTHOR-RECOMMENDED INTERNET RESOURCE}

Cornell University New York State Agricultural Experiment Station website: www.nysaes.cornell.edu/cals/nysaes/bp/shops/greenhouse/pottingmix.cfm 